\title{
Rapid Activation of Dormant Presynaptic Terminals by Phorbol Esters
}

\author{
Chun Yun Chang, ${ }^{1,3}$ Xiaoping Jiang, ${ }^{1}$ Krista L. Moulder, ${ }^{1}$ and Steven Mennerick ${ }^{1,2}$ \\ Departments of ${ }^{1}$ Psychiatry and ${ }^{2}$ Anatomy and Neurobiology, and ${ }^{3}$ Graduate Program in Developmental Biology, Washington University School of \\ Medicine, St. Louis, Missouri 63110
}

\begin{abstract}
Presynaptic stimulation stochastically recruits transmission according to the release probability $\left(P_{\mathrm{r}}\right)$ of synapses. The majority of central synapses have relatively low $P_{\mathrm{r}}$, which includes synapses that are completely quiescent presynaptically. The presence of presynaptically dormant versus active terminals presumably increases synaptic malleability when conditions demand synaptic strengthening or weakening, perhaps by triggering second messenger signals. However, whether modulator-mediated potentiation involves recruitment of transmission from dormant terminals remains unclear. Here, by combining electrophysiological and fluorescence imaging approaches, we uncovered rapid presynaptic awakening by select synaptic modulators. A phorbol ester phorbol 12,13-dibutyrate (PDBu) (a diacylglycerol analog), but not forskolin (an adenylyl cyclase activator) or elevated extracellular calcium, recruited neurotransmission from presynaptically dormant synapses. This effect was not dependent on protein kinase $\mathrm{C}$ activation. After PDBu-induced awakening, these previously dormant terminals had a synaptic $P_{\mathrm{r}}$ spectrum similar to basally active synapses naive to PDBu treatment. Dormant terminals did not seem to have properties of nascent or immature synapses, judged by NR2B NMDAR (NMDA receptor) receptor subunit contribution after PDBu-stimulated awakening. Strikingly, synapses rendered inactive by prolonged depolarization, unlike basally dormant synapses, were not awakened by PDBu. These results suggest that the initial release competence of synapses can dictate the acute response to second messenger modulation, and the results suggest multiple pathways to presynaptic dormancy and awakening.
\end{abstract}

\section{Introduction}

Presynaptic modulators often act by altering the probability of transmitter release $\left(P_{\mathrm{r}}\right)$, which is heterogeneous across the population of presynaptic terminals of a neuron (Hessler et al., 1993; Rosenmund et al., 1993; Murthy et al., 1997). Conventionally, measuring synaptic $P_{\mathrm{r}}$ is accomplished by electrophysiological or optical assessments. Both approaches are biased toward sampling the responses of synapses with higher synaptic $P_{\mathrm{r}}$. Populations of synapses with extremely low $P_{\mathrm{r}}$ or completely dormant presynaptic terminals are more difficult to detect, despite evidence for such populations (Tong et al., 1996; Murthy et al., 1997; Ma et al., 1999; Moulder et al., 2004; Slutsky et al., 2004). Whether these populations are subject to presynaptic modulation by second messenger systems has only begun to be investigated.

There is good evidence that prolonged activation of cAMP/ PKA (protein kinase A) pathways by forskolin (FSK) awakens presynaptically dormant synapses (Chavis et al., 1998; Ma et al., 1999; Moulder et al., 2008). At least in some systems, this presyn-

Received March 7, 2010; revised May 24, 2010; accepted June 16, 2010.

This work was supported by National Institutes of Health Grants MH78823, NS54174 (S.M.), and DA018109 (K.L.M.). We are grateful to William Dribben for kindly sharing equipment; Peter Lukasiewicz, Jim Huettner, and Aaron DiAntonio for guidance and encouragement; Larry Eisenman and Patricio Rojas for sharing and discussing the Igor procedure codes; Ann Benz and Amanda Taylor for help in preparation of the cultures; and laboratory members for critical discussion.

Correspondence should be addressed to Steven Mennerick, Department of Psychiatry, Washington University School of Medicine, 660 South Euclid Avenue, Campus Box 8134, St. Louis, M0 63110. E-mail: menneris@psychiatry.wustl.edu.

DOI:10.1523/JNEUROSCI.1159-10.2010

Copyright $\odot 2010$ the authors $\quad 0270-6474 / 10 / 3010048-13 \$ 15.00 / 0$ aptic activation requires protein synthesis (Ma et al., 1999) and takes several hours. However, rapid activation of cAMP signaling does not seem to increase functional terminals (Trudeau et al., 1996), despite apparent enhancement of synaptic output by electrophysiological assessment (Weisskopf et al., 1994; Trudeau et al., 1996; Lonart et al., 1998). Whether rapid potentiation of transmission by other modulators involves awakening dormant synapses is less clear.

Here, we focus on phorbol ester modulation of glutamate transmission. Classically, phorbol ester stimulation activates protein kinase C (PKC) pathways (Blumberg, 1991). However, phorbol esters also bind to other proteins with diacylglycerol-binding domains, including the vesicle priming protein Munc13; activation of Munc13 by phorbol esters promotes vesicle release through this PKC-independent mechanism (Betz et al., 1998; Rhee et al., 2002). Both PKC-dependent and PKC-independent pathways may participate in acute synaptic modulation by phorbol esters (Wierda et al., 2007; Lou et al., 2008). Although the phenomenon of phorbol ester potentiation and its underlying molecular mechanisms have been intensively studied (Brose and Rosenmund, 2002; Silinsky and Searl, 2003), it remains unknown whether phorbol esters potentiate synaptic function in part by acting on dormant synapses.

Using both electrophysiological and imaging assessments, we show that phorbol esters activate preexisting presynaptically dormant synapses in addition to enhancing transmitter output probability at already active synapses. Interestingly, after activation by phorbol 12,13-dibutyrate (PDBu), presynaptic quiescent synapses appear to have heterogeneous $P_{\mathrm{r}}$ that is similar to basal, 
untreated active synapses. Despite its effects on basally dormant synapses, $\mathrm{PDBu}$ does not reactivate presynaptic terminals rendered inactive by sustained depolarization, suggesting multiple independent routes to presynaptic dormancy. In summary, our work increases understanding of the complex effects of phorbol esters on presynaptic function. Among its rapid effects is a restoration of presynaptic function at terminals that do not normally contribute to synaptic transmission.

\section{Materials and Methods}

Cell cultures. Dissociated hippocampal cultures were prepared as previously described (Mennerick et al., 1995; Moulder et al., 2007). Briefly, hippocampi were harvested from Sprague Dawley rats at postnatal days $1-3$ and were dissociated enzymatically using $1 \mathrm{mg} \mathrm{ml}^{-1}$ papain, and then mechanically with a glass pipette. Dissociated neurons were then plated at 100 cells $\mathrm{mm}^{-2}$ for low-density microisland plating (for electrophysiological recording) or $\sim 650$ cells $\mathrm{mm}^{-2}$ for high-density mass cultures (for imaging experiments). For island cultures, culture plates were precoated with $0.15 \%$ agarose and were stamped or sprayed with type I collagen $\left(0.5 \mathrm{mg} \mathrm{ml}^{-1}\right)$ as the microdot substrate. For mass cultures, collagen was spread uniformly across a $25-\mathrm{mm}$-diameter glass coverslip. Culture media consisted of Eagle's minimal essential medium (Invitrogen) supplemented with 5\% heat-inactivated horse serum, 5\% fetal bovine serum, $17 \mathrm{~mm}$ glucose, $400 \mu \mathrm{M}$ glutamine, $50 \mathrm{U} \mathrm{ml}^{-1}$ penicillin, and $50 \mu \mathrm{g} \mathrm{ml}^{-1}$ streptomycin. To suppress glial proliferation, 6.7 $\mu \mathrm{M}$ cytosine arabinoside was added to the cultures 3-4 d after plating. One-half of the culture media was removed and replaced with Neurobasal medium plus B27 supplement $1 \mathrm{~d}$ after antimitotic addition. Cells were used for experiments 9-14 d after plating.

Electrophysiology. Whole-cell recordings were performed with a MultiClamp 700B amplifier and Digidata 1440A acquisition system (Molecular Devices); data were acquired in Clampex 10 (Molecular Devices). Recordings were conducted in extracellular solution (bath) consisting of the following (in mM): $138 \mathrm{NaCl}, 4 \mathrm{KCl}, 2 \mathrm{CaCl}_{2}, 0.01$ glycine, 10 glucose, and 10 HEPES (Invitrogen), pH 7.25. AMPA-sensitive receptor (AMPAR)mediated current was isolated by 50-75 $\mu \mathrm{M}$ D-amino-5-phosphonovaleric acid (D-APV) (Tocris); NMDA-sensitive receptor (NMDAR)-mediated currents were isolated by 1-2 $\mu \mathrm{M}$ 2,3-dioxo-6-nitro-1,2,3,4-tetrahydrobenzo[f] quinoxaline-7-sulfonamide (NBQX) (Tocris). The $400 \mu \mathrm{m}$ kynurenate (for AMPAR current) and $250 \mu \mathrm{M}$ L-amino-5-phosphonovaleric acid (L-APV) (for NMDAR current) (Tocris) were added to reduce receptor desensitization and to minimize access resistance errors associated with the large autaptic currents. During $(5 S, 10 R)-(+)-5$-methyl-10,11-dihydro- $5 H$ dibenzo $[a, d]$ cyclohepten-5,10-imine maleate (MK-801) blocking procedures, L-APV was omitted. Nontransmitter-mediated residual current was evaluated in the presence of $1-2 \mu \mathrm{M}$ NBQX and $75 \mu \mathrm{M}$ D-APV and was subtracted from the evoked EPSCs. Solution was perfused by a gravity-based multibarrel perfusion system at the rate of $0.2 \mathrm{ml} \mathrm{min}^{-1}$ with $<100 \mathrm{~ms}$ complete solution switch. For sucrose-evoked responses, flow rate was increased to $1.6 \mathrm{ml} \mathrm{min}^{-1}$ with $<50 \mathrm{~ms}$ solution switch. Responses to modulators (PDBu and forskolin) were measured immediately after the $2 \mathrm{~min}$ application. Responses to PDBu remained elevated for at least 2 min after modulator removal (supplemental Fig. S2, available at www.jneurosci.org as supplemental material). Electrode pipettes with open tip resistance of 3-5 $\mathrm{M} \Omega$ were filled with the internal pipette solution containing the following (in $\mathrm{mm}$ ): $140 \mathrm{~K}$-gluconate, $4 \mathrm{NaCl}, 0.5 \mathrm{CaCl}_{2}, 10 \mathrm{EGTA}$, and $10 \mathrm{HEPES}, \mathrm{pH} 7.25$. After the whole-cell mode was established, cells were voltage clamped at -70 $\mathrm{mV}$, and EPSCs were evoked by a brief $(1 \mathrm{~ms})$ depolarization to $0 \mathrm{mV}$. Only cells with access resistance of $<11 \mathrm{M} \Omega$, membrane resistance of $>150 \mathrm{M} \Omega$, and leak current of $<200 \mathrm{pA}$ were recorded. Series resistance was compensated by $80 \%$. Leak current was subtracted off-line. Signals were sampled at 5 or $10 \mathrm{kHz}$ and filtered at $2 \mathrm{kHz}$. All recordings were performed at room temperature.

Pharmacological reagents. Unless otherwise specified, reagents were purchased from Sigma-Aldrich. Stock solutions of MK-801 (10 mM; Tocris), NMDA (100 mM), 4-aminopyridine (4-AP) (100 mm), and ifenprodil (3 $\mathrm{mM})$ were dissolved in distilled water and diluted to the indicated concentrations. Stock solutions of bicuculline (50 mM), forskolin (50 mM), 3-[1-[3- (dimethylamino)propyl])-5-methoxyindol-3-yl]-4-(1 H-indol-3-yl)pyrrole-2, 5-dione (Gö6983) (2 or 20 mM), 3- [3-[4-(1-methylindol-3-yl)-2,5-dioxopyrrol-3-yl]indol-1-yl]propylcarbamimidothioate (Ro31-8220) (2 or $20 \mathrm{~mm}$ ), and PDBu ( 1 or $5 \mathrm{~mm}$ ) were made in DMSO and diluted in working solutions. The final concentration of DMSO was never higher than $0.1 \%$. This DMSO concentration had no effect on synaptic transmission (supplemental Fig. S3, available at www.jneurosci.org as supplemental material).

Data analysis. Data were analyzed by Clampfit10 (Molecular Devices) or by customized Igor Pro procedures (Wavemetrics). Residual stimulation-associated capacitive and presynaptic currents in the sample traces in all figures were blanked for clarity; sample traces in Figures 3 and 7 were low-pass filtered with a digital eight-pole Bessel filter at $500 \mathrm{~Hz}$ for clarity. Data fitting was performed by commercially available fitting routines (Clampfit and Igor Pro). All results are presented as mean \pm SEM. Paired or unpaired $t$ test or one-way ANOVA were used for statistic analysis.

Imaging experiments, image acquisition, and analysis. Mass cultures prepared under identical conditions and in parallel with autaptic cultures were used for imaging experiments 11-14 d in vitro. Mass cultures were used because single synapses could be identified much more reliably in this culture preparation than in autaptic cultures. Autaptic synapses tend to nest near the soma and proximal dendrites, and the extensive synaptic overlap makes sampling individual synapses problematic. Before the experiment, the cultures were rinsed with extracellular solution plus $50 \mu \mathrm{M}$ D-APV and $1 \mu \mathrm{M}$ NBQX. Functional synapses were identified by an FM143FX dye loading-based assay (Betz and Bewick, 1992). This assay takes advantage of activity-dependent dye loading in presynaptic terminals whose vesicles actively participate in exo-endocytic cycles. Such presynaptic FM dye loading does not occur in presynaptic terminals that are functionally silent (Rosenmund et al., 2002; Altrock et al., 2003; Moulder et al., 2004, 2006).

To label functional synapses, hyperkalemic extracellular solution (45 $\mathrm{mm}\left[\mathrm{K}^{+}\right]_{\mathrm{o}}$, equimolar substitution for $\mathrm{Na}^{+}$, with $25 \mu \mathrm{M} \mathrm{D}-\mathrm{APV}$ and $1 \mu \mathrm{M}$ NBQX) was used to trigger transmitter release in the presence of FM1$43 \mathrm{FX}$ ( $10 \mu \mathrm{M}$; Invitrogen) for $2 \mathrm{~min}$. This protocol is designed to release all functionally competent vesicles in the recycling pool, regardless of vesicular $P_{\mathrm{r}}$ (Pyle et al., 2000; Harata et al., 2001). The FM1-43FX loading protocol is expected to directly depolarize the membrane potential to approximately $-20 \mathrm{mV}$ (Moulder et al., 2006; Crawford et al., 2009) and therefore promote transmitter release from terminals, independent of presynaptic action potentials. After labeling, Advasep-7 (500 $\mu \mathrm{M}$; CyDex) was added to remove residual dye for another $10 \mathrm{~s}$. Dye labeled cultures were rinsed and fixed in $4 \%$ paraformaldehyde and $0.2 \%$ glutaraldehyde in PBS for 10 min. Fixed cultures were incubated in blocking solution containing $4 \%$ goat serum and $0.05 \%$ Triton X-100 for 15 min. For vesicular glutamate transporter 1 (vGluT-1) staining, the fixed cultures were incubated in primary vGluT-1 antisera (Millipore Bioscience Research Reagents) diluted 1:2000 in blocking solution for $3 \mathrm{~h}$. Alexa Fluor 647-conjugated anti-guinea pig antisera (1:500; Invitrogen) were used to visualize vGluT-1 labeling. Cultures on glass were mounted on a microscope slide with Fluoromount-G (Southern Biotechnology). Experimental conditions for each slide were coded before subsequent image acquisition and analysis, and the experimenter was kept naive to experimental conditions through the data acquisition and analysis procedures.

All images were acquired under a $60 \times$ oil objective by a $\mathrm{C} 1$ scanning laser coupled to a Nikon TE300 inverted microscope (Nikon). Acquisition gain setting, scanning rate, and $Z$-stack setting were identical across the acquired fields within an experiment. Data analysis was performed in MetaMorph (Molecular Devices). Fluorescence images were acquired sequentially from the vGluT-1 and FM1-43FX channels. Fields were selected on the basis of exhibiting discrete vGluT-1-immunoreactive puncta (Moulder et al., 2010). Ten regions of interest in each field, corresponding to vGluT-1-immunoreactive puncta, were selected without reference to the FM1-43FX image. The FM1-43FX image was then superimposed and independently thresholded. To distinguish active from inactive synapses, we used a relative FM1-43FX thresholded area cutoff. The thresholded FM1-43FX area of active synapses was required to be $>12.5 \%$ of the thresholded vGluT-1 area of the same punctum. This 
A

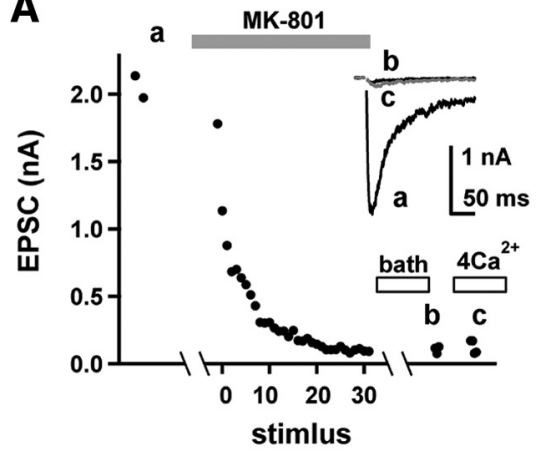

D1

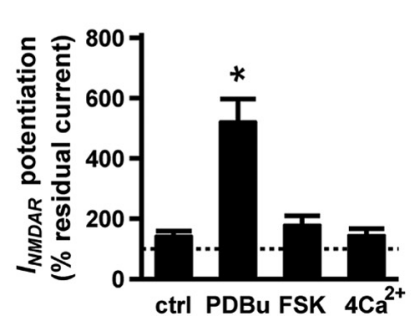

B

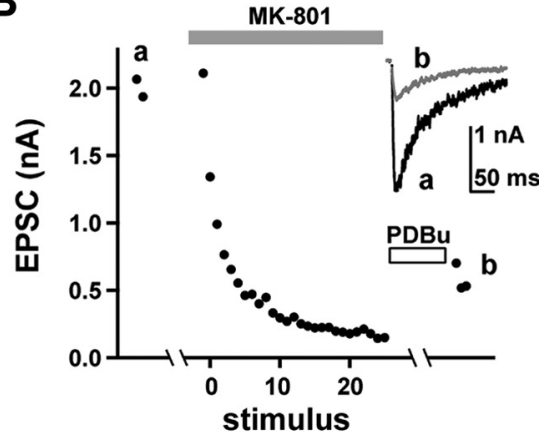

C

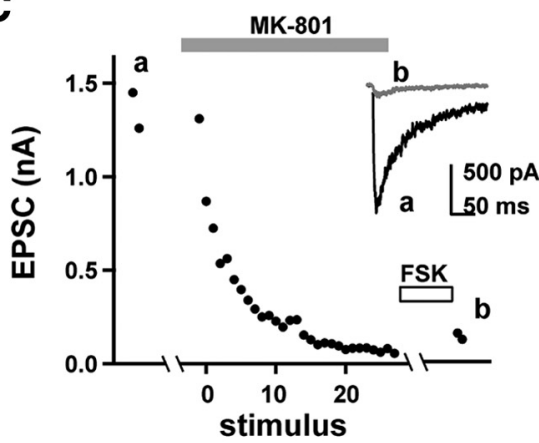

2

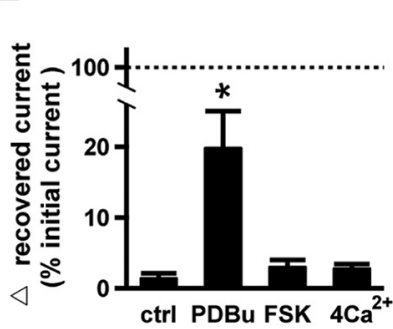

E1

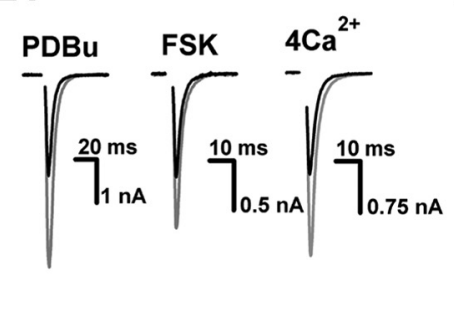

2

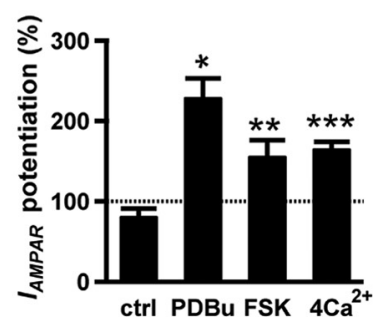

Figure 1. Modulators differentially potentiate NMDAR-mediated EPSCs after MK-801 block of synaptic NMDARs. A, Example of progressive MK-801 block during evoked stimulation and subsequent recovery of $/_{\text {NMDAR}}$. Synaptic receptor blockade was accomplished by delivering low-frequency presynaptic stimulation $(0.1 \mathrm{~Hz})$ in the presence of MK-801 $(5 \mu \mathrm{M})$. The gray bar indicates the time period when MK-801 was applied; the open bars show application of regular extracellular saline solution (bath) or of elevated $\mathrm{Ca}^{2+}\left(4 \mathrm{Ca}^{2+}\right)$. The recovered $I_{\text {NMDAR }}$ in regular external bath or $4 \mathrm{mM} \mathrm{Ca}{ }^{2+}$ was sampled 2-3 min after MK-801 wash off. The amplitude of $I_{\text {NMDAR }}$ is plotted against the stimulus episode. Inset, Superimposed initial (a), the basal recovered $I_{\text {NMDAR }}(\mathrm{b})$, and the recovered current in $4 \mathrm{~mm} \mathrm{Ca}{ }^{2+}$ (c) shown from the corresponding time point in the amplitude-stimulus plot. $\boldsymbol{B}$, C, Similar to $\boldsymbol{A}$, with PDBu $(1 \mu \mathrm{M})(\boldsymbol{B})$ or FSK (50 $\left.\mu \mathrm{m}\right)(\boldsymbol{C})$ applied during the 2 min

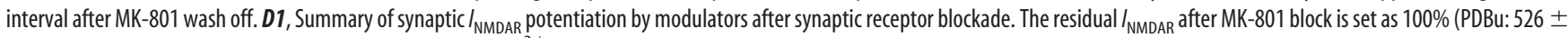
$71 \%, n=10,{ }^{*} p<0.00007 ;$ FSK: $184 \pm 25 \%, n=7 ; 4 \mathrm{mM} \mathrm{Ca}^{2+}: 150 \pm 17 \%, n=8$; statistical comparison is with the control: $\left.147 \pm 12 \%, n=8\right)$. D2, Comparison of the percentage $I_{\text {NMDAR }}$ recovery $\left(\Delta\right.$ recovered current $=$ recovered current - residual current) after modulator treatment. The $\Delta$ recovered $I_{\text {NMDAR }}$ is normalized to the initial $I_{\text {NMDAR }}$. The initial $I_{\text {NMDAR }}$ is set to $100 \%$ (PDBu: $20 \pm 5 \%, n=10,{ }^{*} p<0.005 ;$ FSK: $3.2 \pm 0.8 \%, n=7 ; 4 \mathrm{~mm} \mathrm{Ca}{ }^{2+}: 3.0 \pm 0.4 \%, n=8$; statistical comparison is with the control: $\left.1.6 \pm 0.5 \%, n=8\right) . E$, Synaptic NMDAR blockade did not affect $I_{\text {AMPAR }}$ potentiation by modulators. E1, Sample traces of $I_{\text {AMPAR }}$ in $4 \mathrm{mM} \mathrm{Ca}{ }^{2+}$, PDBu, and FSK (gray); traces are superimposed on their corresponding initial EPSC (black). The percentage potentiation of EPSCS by modulators is summarized in E2 (PDBu: $230 \pm 23 \%$ of initial $I_{\text {AMPAR }}, n=10,{ }^{*} p<0.0002 ;$ FSK: $157 \pm 19 \%$ of the initial $I_{\text {AMPAR }}, n=7$, ${ }^{* *} p<$ $0.02 ; 4 \mathrm{mM} \mathrm{Ca}{ }^{2+}: 163 \pm 8 \%$ of the initial $I_{\text {AMPAR }^{\prime}} n=7,{ }^{* * *} p<0.002$; all statistics are compared with the control: $\left.83 \pm 8 \%, n=8\right)$. Error bars indicate SEM.

criterion was chosen because the nonthresholded FM1-43FX labeling intensity of 51 synapses identified as inactive by this criterion in our data set (five experiments) was not significantly different from surrounding cellular background fluorescence near the same synapses $(p>0.1)$. The use of a percentage area criterion guarded against artifactual assignment of small synapses to the inactive group, but an activity criterion based on a fixed-area cutoff of 10 thresholded pixels of FM1-43FX staining (Moulder et al., 2006) yielded similar results (see Results). For the present experiments, 250 vGluT-1-immunoreactive synapses from five independent experiments (10 puncta from each of five fields in each experiment) were analyzed in each condition. Additional evidence for presynaptically inactive synapses is the bimodal distribution of FM1-43FX fluorescence intensity of vGluT-1-positive terminals when hundreds of terminals from a single experiment are examined (Moulder et al., 2006). One component of the distribution is indistinguishable from background fluorescence and thus represents the population of inactive terminals. A second, broader, nonzero component represents active terminals (Moulder et al., 2006).

\section{Results}

PDBu recovers postblock NMDAR-mediated current

We first tested whether synaptic modulators could potentiate transmitter output from synapses with basally low release activity. To selectively eliminate neurotransmission from active synapses, we took advantage of an irreversible use-dependent NMDAR antagonist MK-801 (Huettner and Bean, 1988). At lowfrequency stimulation $(0.1 \mathrm{~Hz}), \mathrm{MK}-801$ blocks NMDAR only at synapses that release glutamate in response to the stimulation (Hessler et al., 1993; Rosenmund et al., 1993). Consequently, the synaptic NMDAR remaining functional after MK-801 blocking trials should represent the receptors residing at synapses that are the least active during the stimulation. We examined whether modulator application after synaptic block of NMDAR current $\left(I_{\mathrm{NMDAR}}\right)$ causes recovery of synaptic $I_{\mathrm{NMDAR}}$. The recovered $I_{\mathrm{NMDAR}}$ is presumably mediated by the receptors that remain functionally intact after MK-801 block and therefore represent the transmitter output from synapses that release glutamate only after modulator treatment.

In the presence of MK-801 (5 $\mu \mathrm{M}), 25-35$ action potential stimuli at $0.1 \mathrm{~Hz}$ depressed synaptic $I_{\mathrm{NMDAR}}$ to $\sim 3 \%$ of the initial $I_{\mathrm{NMDAR}}\left(3 \pm 0.4 \%\right.$ of the initial $\left.I_{\mathrm{NMDAR}} ; n=8\right)$ (Fig. $\left.1 \mathrm{~A}\right)$. Isolated AMPAR current $\left(I_{\mathrm{AMPAR}}\right)$ and the $I_{\mathrm{AMPAR}}$ paired-pulse ratio (PPR) (second EPSC/first EPSC; 50 ms interpulse interval), however, was minimally affected by MK-801 application $\left(I_{\mathrm{AMPAR}}\right.$ amplitude after the MK-801 blocking protocol, $83 \pm 7 \%$ of the initial $I_{\mathrm{AMPAR}} ; p=0.06$; baseline PPR, $0.71 \pm 12 \%$; PPR after MK-801 blocking, $0.66 \pm 6 \% ; p=0.09 ; n=8)$. The insignificant trend toward reduced $I_{\mathrm{AMPAR}}$ likely resulted from weak, variable time-dependent rundown. These results indicate that MK-801 selectively antagonizes NMDARs; blocking synaptic NMDARs did not detectably change presynaptic release properties. There was very little recovery of $I_{\mathrm{NMDAR}} 2-3 \mathrm{~min}$ after MK-801 removal (Fig. 1A,D2). The slight increase of $I_{\mathrm{NMDAR}}$ may reflect minor dissociation of MK- 801 from the receptors because of the preceding trials of depolarization applied to sample the residual current after MK-801 block (Huettner and Bean, 1988). Alternatively, the 
weak recovery of residual current might represent a small contribution from receptor diffusion from perisynaptic to synaptic sites (Tovar and Westbrook, 2002). Regardless of the causes of the weak recovery, the small fraction of current increase under control conditions indicated that MK-801 block was relatively irreversible within the time interval of our experiments (Huettner and Bean, 1988). Applying similar blocking trials to neurons in 4 $\mathrm{mM}\left[\mathrm{Ca}^{2+}\right]$, which was designed to raise vesicular $P_{\mathrm{r}}$ at active terminals, did not further reduce the residual current (blocking in $4 \mathrm{mM} \mathrm{Ca}^{2+}, 3.2 \pm 1.0 \%$ to the initial $I_{\mathrm{NMDAR}} ; n=8$; blocking in $\left.2 \mathrm{mM} \mathrm{Ca}^{2+}, 4.6 \pm 0.8 \% ; p=0.32 ; n=9\right)$, suggesting application of MK- 801 in $2 \mathrm{mM} \mathrm{Ca}^{2+}$ using our stimulation protocol was sufficient to block virtually all active synapses. Together, these results confirm that our protocol asymptotically blocked NMDARs at the population of presynaptically active terminals.

After similar blocking trials with MK-801, application of $\mathrm{PDBu}$, as previously documented, strongly potentiated $I_{\mathrm{AMPAR}}$ (Fig. 1E1,E2). PDBu effects on AMPAR-mediated EPSCs have previously been shown to be primarily through presynaptic mechanisms (Malenka et al., 1986; Hori et al., 1999) (but see Carroll et al., 1998). Interestingly, $\mathrm{PDBu}$ also promoted a strong recovery of synaptic $I_{\mathrm{NMDAR}}$ after MK-801 block (Fig. $1 B, D 1, D 2)$. PDBu resulted in approximately fivefold potentiation of the residual $I_{\mathrm{NMDAR}}$ after MK-801 block (Fig. 1D1), and the net current recovery was $\sim 20 \%$ of the initial current before receptor block (Fig. 1D2).

cAMP increases awakening of dormant presynaptic terminals, but the effect is slow to develop (Ma et al., 1999; Yao et al., 2006). We used acutely applied FSK to determine whether rapid cAMP increases, similar to rapid PDBu effects, recruit release from synapses that are not blocked by MK-801. As a presynaptic comparator, we also examined the effect of elevated extracellular $\mathrm{Ca}^{2+}$ on residual $I_{\mathrm{NMDAR}}$. As expected, FSK application robustly potentiated $I_{\mathrm{AMPAR}}$ (Fig. 1 E1,E2). However, in contrast to PDBu, acute FSK application after MK-801 block resulted in only a minimal recovery of postblock synaptic $I_{\mathrm{NMDAR}}$ (Fig. 1C,D1,D2). Evoked EPSCs in $4 \mathrm{mM} \mathrm{Ca}^{2+}$, which is expected to increase vesicular $P_{\mathrm{r}}$ at active terminals but not to awaken dormant synapses, increased $I_{\mathrm{AMPAR}}$ to $\sim 1.5$-fold (Fig. $1 E 1, E 2$ ), but again this manipulation caused only a minimal increase of $I_{\text {NMDAR }}$ after MK801 block (Fig. 1A,D1,D2). The residual current potentiation and the ratio of current recovery to the initial current before MK-801 block were similar in FSK and $4 \mathrm{mM} \mathrm{Ca}^{2+}$ (Fig. $1 D 1, D 2)$, suggesting that acute FSK, similar to elevated $\mathrm{Ca}^{2+}$, did not significantly recruit transmission from synapses protected from MK-801 block. The minimal recovery of $I_{\mathrm{NMDAR}}$ in $4 \mathrm{mM}$ $\mathrm{Ca}^{2+}$ after MK-801 block, which potentiated $I_{\text {AMPAR }}$, also indicates that potentiation of residual $I_{\mathrm{NMDAR}}$ did not result from transmitter spillover to the receptor domains that were not previously exposed to transmitter.

To confirm further that the recovered $I_{\text {NMDAR }}$ after $\mathrm{PDBu}$ potentiation was not mediated by residual receptors that were not completely blocked at the initially active terminals, we also examined the effect of PDBu on $I_{\text {NMDAR }}$ recovery after MK-801 block of synaptic current in elevated $\mathrm{Ca}^{2+}(4 \mathrm{mM})$. Blocking receptors with $\mathrm{MK}-801$ in higher $\mathrm{Ca}^{2+}$, which raises overall vesicular and synaptic $P_{\mathrm{r}}$ at active terminals while block is proceeding, ensures even more complete receptor block, including extrasynaptic receptors that might be recruited only under high vesicular $P_{\mathrm{r}}$ conditions. After receptor block in elevated $\mathrm{Ca}^{2+}, \mathrm{PDBu}$ still significantly promoted $I_{\mathrm{NMDAR}}$ recovery (PDBu potentiation, $515 \pm 52 \%$ of residual current; $n=8$ ). This result confirms that $\mathrm{PDBu}$-dependent recovery of $I_{\mathrm{NMDAR}}$ was not caused by incomplete receptor block at the terminals in combination with transmitter spillover after potentiation. We hypothesized that PDBuinduced $I_{\text {NMDAR }}$ recovery after MK-801 block represents a unique presynaptic effect of $\mathrm{PDBu}$ potentiation at initially dormant synapses.

Although these experiments help exclude incomplete MK-801 block because of low vesicular $P_{r}$, they do not account for the possibility that NMDA receptors remain unblocked because of action potential propagation failures into terminals during stimulation. PDBu might recover synaptic $I_{\mathrm{NMDAR}}$ as a result of increased excitability in axon branches that normally fail to transmit action potentials. We have previously shown that action potential invasion is reliable at autaptic synapses under normal conditions, and manipulations designed to increase axon excitability do not significantly alter autaptic output (He et al., 2002). Nevertheless, we performed additional tests for the present work. First, we tested whether increasing presynaptic stimulus duration recruits larger synaptic responses. We found no significant change in EPSC amplitude with presynaptic stimulus durations of 1, 2, or $3 \mathrm{~ms}$ (supplemental Fig. S1A, available at www. jneurosci.org as supplemental material). Furthermore, wider stimuli did not recover more synaptic $I_{\mathrm{NMDAR}}$ after the MK-801 blocking protocol (supplemental Fig. $\mathrm{S} 1 \mathrm{~B}$, available at www. jneurosci.org as supplemental material). As another test of complete MK-801 block, we applied 4-AP (100 $\mu \mathrm{M})$ after the MK-801 blocking protocol to artificially enhance excitability of unclamped axonal regions. We observed no significant $I_{\text {NMDAR }}$ recovery after MK-801 block (supplemental Fig. S1C, available at www.jneurosci.org as supplemental material), unlike our observations with $\mathrm{PDBu}$. Additional evidence that changes in excitability do not explain the PDBu effect come in experiments below in which we used secretagogues (e.g., hypertonic challenge and direct depolarization) designed to circumvent action potential propagation entirely. Together, these results all suggest that $\mathrm{PDBu}$-induced excitability changes do not account for the observation of $I_{\mathrm{NMDAR}}$ recovery after MK-801 block.

\section{Modulators equally enhance $I_{\mathrm{AMPAR}}$ and $I_{\mathrm{NMDAR}}$}

To test our hypothesized explanation of PDBu induced current recovery after MK-801 block, we next performed various tests of a postsynaptic explanation for the PDBu-induced $I_{\text {NMDAR }}$ recovery. Differential effects of PDBu and FSK on post-MK-801, residual $I_{\text {NMDAR }}$ may result from preferential potentiation of specific postsynaptic receptors (i.e., PDBu preferentially potentiated $I_{\mathrm{NMDAR}}$, or FSK preferentially potentiated $\left.I_{\mathrm{AMPAR}}\right)$. In mature hippocampal neurons, AMPARs and NMDARs are colocalized in excitatory terminals (Forsythe and Westbrook, 1988; Bekkers and Stevens, 1989); pure presynaptic potentiation typically affects AMPAR- and NMDAR-mediated EPSCs in parallel (Perkel and Nicoll, 1993; Tong and Jahr, 1994). If PDBu and FSK preferentially modulate NMDARs or AMPARs, we would expect that PDBu and FSK should differentially affect EPSCs mediated by NMDARs versus EPSCs mediated by AMPARs. We measured dual-component EPSCs before and after modulator application in the absence of MK-801 (Fig. 2 A). We defined the amplitude of the AMPAR component of the EPSC as the current peak within $10 \mathrm{~ms}$ after stimulation was initiated, whereas the amplitude of $I_{\text {NMDAR }}$ was denoted as the averaged current within a $5 \mathrm{~ms}$ bin 35 ms after stimulation initiation (Tsien et al., 1996; Myme et al., 2003).

Application of PDBu potentiated $I_{\mathrm{AMPAR}}$ and $I_{\mathrm{NMDAR}}$ in parallel, with no distinguishable difference in the degree of potentiation (Fig. 2A, left; $B$, left panel). This result is consistent with the 
idea that acute PDBu treatment has primarily a presynaptic action. After acute incubation with FSK, potentiation in $I_{\mathrm{AMPAR}}$ was also indistinguishable from $I_{\mathrm{NMDAR}}$ potentiation (Fig. $2 \mathrm{~A}$, right; $B$, right panel). Thus, FSK treatment also resulted in an equal enhancement of $I_{\mathrm{AMPAR}}$ and $I_{\mathrm{NMDAR}}$. These results showed that, with brief incubation, PDBu and FSK did not preferentially augment current mediated by specific postsynaptic receptors and therefore suggest a presynaptic effect of EPSC potentiation.

Increases in open probability $\left(P_{\mathrm{o}}\right)$ of NMDARs have been reported with phorbol ester stimulation and/or PKC activation (Durand et al., 1992; Xiong et al., 1998; Lan et al., 2001). An increase in $P_{\mathrm{o}}$ might be expected to result in nonparallel modulation of AMPAR and NMDAR EPSC components. Therefore, the results in Figure 2 argue against a PDBu effect on $P_{\mathrm{o}}$ under our conditions. In addition, if PDBu increases $P_{\mathrm{o}}$ of NMDARs and thereby contributes to the $I_{\mathrm{NMDAR}}$ recovery after MK-801 block, we would also expect that $\mathrm{PDBu}$ should retard the decay of NMDAR-mediated EPSCs (Jahr, 1992). However, we found that, in the presence of PDBu, the weighted time constant of NMDARmediated EPSCs was $178 \pm 10 \mathrm{~ms}$, which was not significantly different from the weighted decay constant in the baseline (164 \pm $28 \mathrm{~ms} ; n=7 ; p=0.86)$. Therefore, PDBu-mediated recovery of $I_{\text {NMDAR }}$ does not result from increased $P_{\mathrm{o}}$ of NMDARs under our conditions.

\section{PDBu does not increase surface NMDARs}

Work from others has shown that prolonged treatment with phorbol esters promotes surface NMDAR insertion and receptor assembly in hippocampal neurons (Lan et al., 2001; Scott et al., 2001). We next explored whether acute treatment with PDBu in our system leads to detectable increases in surface NMDARs that might cause $I_{\text {NMDAR }}$ recovery after MK-801 block. To test whether PDBu increased surface NMDARs, we blocked whole-cell NMDARs by applying MK-801 $(20 \mu \mathrm{M})$ in the presence of the exogenous agonist NMDA ( $1 \mathrm{mM})$ and examined the increases of the whole-cell $I_{\text {NMDAR }}$ after PDBu treatment. Wholecell blockade of NMDARs depressed NMDA-evoked current to $\sim 4 \%$ of the initial current $(4.3 \pm 1.8 \% ; n=6$ ) (Fig. $3 A$, top panel). Whole-cell blockade of NMDARs affected neither evoked synaptic $I_{\text {AMPAR }}$ $(104 \pm 10 \%$ of baseline; $n=6)$ nor PPR measured by $I_{\mathrm{AMPAR}}$ (baseline PPR, $0.89 \pm 15 \%$; PPR after whole-cell block, $0.78 \pm 17 \% ; p=0.32$ ), suggesting that blocking whole-cell NMDARs did not affect presynaptic transmitter output. Two minutes after MK-801 wash-off, residual whole-cell responses to NMDA were slightly increased (Fig. 3A, top panel; $C$, left). The slight increase of whole-cell $I_{\mathrm{N}^{-}}$ MDAR may result from weak unblocking of antagonist from the receptors during the preceding trial of NMDA application, which measured the residual current after whole-cell MK-801 block (Huettner and Bean, 1988). Acute PDBu application after whole-cell receptor blockade resulted in minimal increases in the NMDA-evoked whole-cell current (Fig. 3A, bottom panel). The
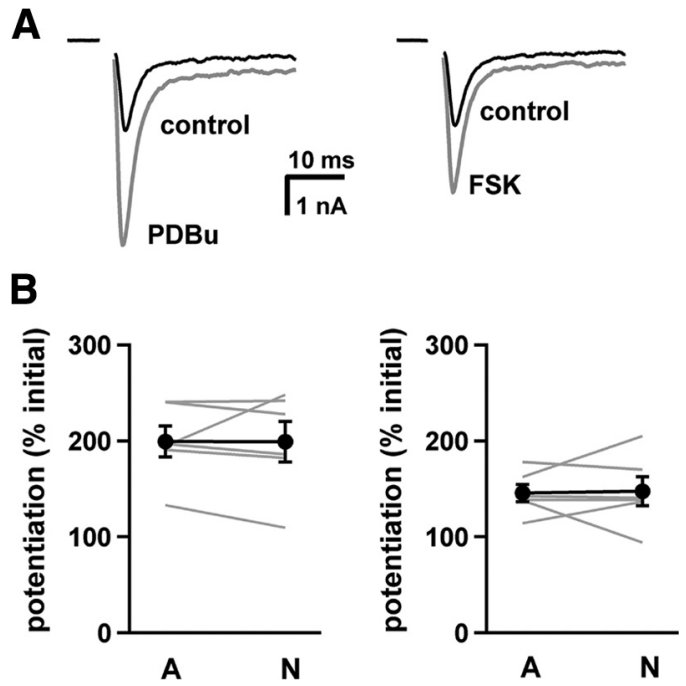

Figure 2. Modulators equally potentiate AMPAR- and NMDAR-mediated EPSCs. $\boldsymbol{A}$, The effect of modulators on AMPAR and NMDAR EPSCS was examined by comparing AMPAR and NMDAR components of EPSCs before and after 2 min of modulator application. Examples of EPSC in PDBu (left panel; gray) or FSK (right panel; gray) are superimposed on the respective baseline EPSCs (black). B, Summary of potentiation of AMPAR (A) or NMDAR (N) components by $\mathrm{PDBu}$ (left) and FSK (right). The gray lines represent responses from individual cells. For PDBu, $I_{\text {AMPAR }}, 199 \pm 16 \%$ of baseline; $I_{\text {NMDAR }}, 199 \pm 21 \%$ of baseline; $n=6 ; p=0.99$. For FSK, $I_{\text {AMPAR }^{\prime}} 145 \pm 9 \%$ of baseline; $I_{\text {NMDAR}^{\prime}} 147 \pm 15 \%$ of baseline; $n=6 ; p=0.88$. Error bars indicate SEM.

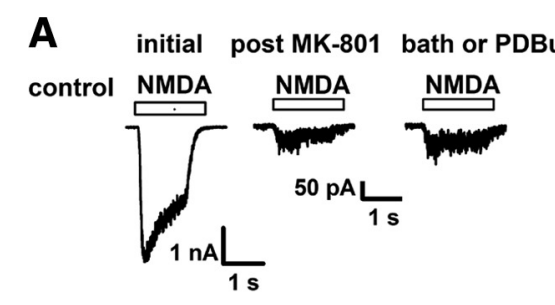

PDBu

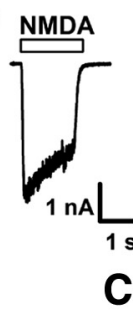

B

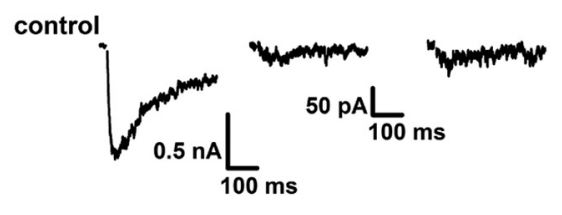

PDBu

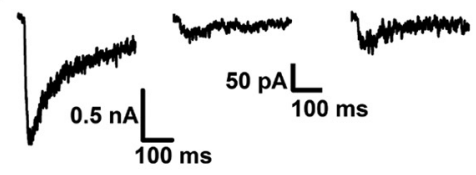

Figure 3. Acute PDBu application does not increase surface NMDARs. $\boldsymbol{A}$, After whole-cell blockade of NMDARs, PDBu caused minimal recovery of whole-cell $I_{\text {NMDAR }}$. Sample traces of time-dependent $(\boldsymbol{A}$, top) or PDBu-dependent $(\boldsymbol{A}$, bottom) effect on whole-cell $/_{\text {NMDAR }}$ after blocking surface NMDARs. $\boldsymbol{B}$, Similar to $\boldsymbol{A}$. The comparison of time-dependent effect and the effect of PDBu on synaptic IMDAR $_{\text {NAter whole-cell NMDAR blockade. C, Quantification of residual whole-cell and synaptic I }}$ NMDAR after control or PDBu treatment after surface receptor blockade. The peak currents are normalized to the initial current before MK- 801 block (whole-cell current: $5.8 \pm 2.5 \%$ in control, $n=6 ; 6.2 \pm 2.2 \%$ in PDBu, $n=6 ; p=0.58$; synaptic current: $5.2 \pm 1.2 \%$ in control, $n=6 ; 7 \pm 2.5 \%$ in $\mathrm{PDBu}, n=6 ; p=0.59$ ). Error bars indicate SEM.

mild increases in NMDA-evoked current in PDBu was indistinguishable from that in control (Fig. 3C, left), suggesting that acute application of PDBu did not significantly increase total NMDARs at the cell surface. 
A1

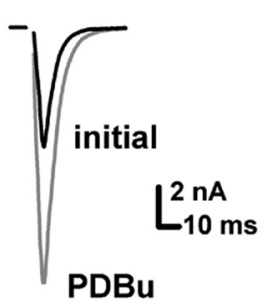

B1

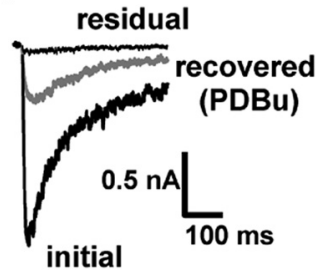

2

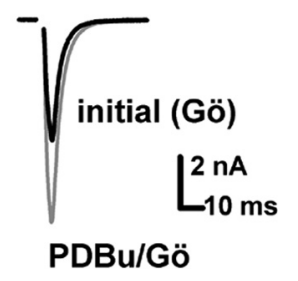

2

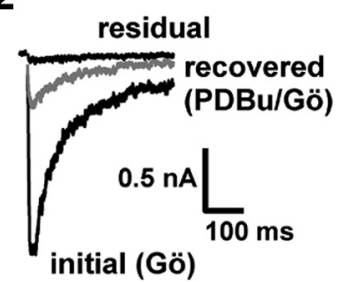

Figure 4. PKC inhibition does not prevent $I_{\text {NMDAR }}$ recovery by PDBu. $\boldsymbol{A}$, PDBu effectively potentiated $I_{\text {AMPAR }}(\boldsymbol{A} 1)$ and robustly recovered $I_{\text {NMDAR }}($ B1) after MK-801 block. A1, Sample trace of $I_{\text {AMPAR }}$ potentiation after PDBu treatment from the same cell is superimposed on the initial current. B1, PDBu-recovered $/_{\text {NMDAR }}$ is superimposed on the initial (before blocking) and the postblock residual current. $\boldsymbol{A 2}$, B2, Similar to $\boldsymbol{A} \mathbf{1}$ and $\boldsymbol{B 1}$, PKC inhibitor Gö6983 (2 $\mu \mathrm{m}$ ) was present before and during the experiments. Average PDBu effect on $I_{\text {AMPAR }}$ potentiation in the absence (PDBu) or presence (PDBu/Gö) of PKC inhibitor: $192.3 \pm 23.5 \%$ in PDBu, $n=9$; $147.2 \pm 19.1 \%$ in PDBu/Gö6983, $n=11 ; p=0.15$. Average effects of PDBu from the experiment in $B$ are given as percentage of initial current before MK-801 block: $17.6 \pm 4.7 \%$ in PDBu, $n=9 ; 15.4 \pm 2.9 \%$ in PDBu/Gö6983, $n=11 ; p=0.7$.

To test whether $\mathrm{PDBu}$ selectively increases synaptic NMDARs, we examined the effect of PDBu on the synaptic $I_{\text {NMDAR }}$ after whole-cell blockade of NMDARs. Consistent with the results from sampling whole-cell NMDA responses, we found that, after whole-cell blockade of NMDARs, PDBu application did not significantly increase synaptic $I_{\mathrm{NMDAR}}$ compared with control cells (Fig. $3 B, C$, right), despite strong potentiation measured in $I_{\text {AMPAR }}(225 \pm 32 \%$ of baseline; $n=6 ; p<0.04)$. These results therefore suggest that acute treatment of $\mathrm{PDBu}$ did not lead to significant increases of NMDARs in postsynaptic sites. Together, our results suggest that acute application of PDBu does not detectably increase surface NMDARs.

\section{PDBu-mediated recovery of $I_{\mathrm{NMDAR}}$ is PKC independent}

Under certain conditions, phorbol esters enhance the clustering, movement, and channel gating of NMDARs (Durand et al., 1992; Kutsuwada et al., 1992; Lu et al., 2000; Lan et al., 2001; Scott et al., 2001; Groc et al., 2004), and these effects are PKC dependent (Durand et al., 1992; Xiong et al., 1998; Lu et al., 2000; Lan et al., 2001; Scott et al., 2001; Fong et al., 2002). Presynaptically, phorbol esters act at least partly through PKC-independent mechanisms to potentiate transmission (Searl and Silinsky, 1998; Rhee et al., 2002; Lou et al., 2008). To distinguish whether postsynaptic $\mathrm{PKC}$ activation by PDBu results in NMDAR modification, which in turn leads to the current recovery after MK-801 block, we evaluated the action of PDBu after pharmacological inhibition of PKC activity. Because PKC activity is present in both presynaptic and postsynaptic compartments, we first tested PKCindependent presynaptic potentiation of PDBu. We measured PDBu potentiation of $I_{\mathrm{AMPAR}}$ in the presence a PKC inhibitor. We used the broad-spectrum PKC inhibitor Gö6983 to block PKC activation by PDBu. The pharmacological activity of Gö6983 was verified by two independent assays in hippocampal excitatory autapses (Chang and Mennerick, 2010). These control experi-

ments were performed concurrently with experiments in the present work and showed that Gö6983 inhibits two previously documented PKC-dependent phorbol ester effects: phorbol ester-induced speeding of vesicle replenishment after depletion (Stevens and Sullivan, 1998) and phorbol ester-induced occlusion of $\mathrm{G}_{\mathrm{i} / \mathrm{o}} \mathrm{G}$-protein-mediated synaptic depression (Zamponi et al., 1997).

Application of Gö6983 (2 $\mu \mathrm{M})$ alone did not alter evoked AMPAR-mediated EPSCs (control, $5.06 \pm 0.98 \mathrm{nA}, n=9$; Gö6983, $4.82 \pm 0.78 \mathrm{nA}, n=10 ; p=0.85)$. Preincubation of Gö6983 did not prevent subsequent $\mathrm{PDBu}$ potentiation of $I_{\mathrm{AMPAR}}$ (Fig. 4A1,A2), consistent with an important role of PKC-independent mechanisms in phorbol ester potentiation (Rhee et al., 2002).

Having confirmed that PDBu effectively potentiated presynaptic neurotransmission in the absence of PKC activation, we next asked whether PKC inhibition, which should also prevent postsynaptic modification of NMDARs, compromises current recovery by PDBu. After MK-801 block of synaptic NMDARs, $\mathrm{PKC}$ inhibition did not prevent $\mathrm{PDBu}$-mediated recovery of synaptic $I_{\text {NMDAR }}$ (Fig. 4B2) compared with PDBu alone. Inhibiting PKC activity by another class of PKC inhibitor, Ro31-8220 (2 $\mu \mathrm{M})$, yielded a similar result $\left(I_{\mathrm{AMPAR}}\right.$ potentiation: $172.1 \pm 12.8 \%$ in PDBu/Ro31-8220, $n=11 ; 246.1 \pm 36.1 \%$ in PDBu, $n=10$; $p=0.07 ; I_{\mathrm{NMDAR}}$ recovery: $20.5 \pm 4.7 \%$ in $\mathrm{PDBu} / \mathrm{Ro} 31-8220$, $n=11 ; 32.5 \pm 7.1 \%$ in PDBu, $n=10 ; p=0.17)$. These results suggest that PKC-dependent modulation of postsynaptic NMDARs does not contribute strongly to the recovery of $I_{\text {NMDAR }}$. Together, these results suggest that the enhancement by PDBu of synaptic strength at terminals with very low synaptic $P_{\mathrm{r}}$ or dormant terminals is mainly $\mathrm{PKC}$ independent.

\section{PDBu potentiation and the readily releasable vesicle pool}

If our hypothesis is correct that PDBu recovers $I_{\mathrm{NMDAR}}$ by presynaptically resurrecting functionally dormant synapses, this effect should also be evident as increases in the number of readily releasable vesicles, the vesicle pool that is docked and primed at presynaptic active zones. Readily releasable vesicle pool (RRP) size is often measured as hypertonic sucrose-evoked AMPA EPSCs (Rosenmund and Stevens, 1996; Schikorski and Stevens, 2001). Hypertonicity-evoked release also circumvents potential PDBu effects on action potential propagation or waveform, since this secretagogue bypasses action potential propagation and $\mathrm{Ca}^{2+}$ influx (Rosenmund and Stevens, 1996). We used $0.75 \mathrm{M}$ sucrose to ensure a saturated rate of vesicle release (Rosenmund and Stevens, 1996; Basu et al., 2007; Wierda et al., 2007). In our cultures, PDBu treatment led to an $\sim 25 \%$ increase in the RRP (Fig. 5A), quantitatively consistent with the degree of $I_{\mathrm{NMDAR}}$ recovery observed in Figure $1 D 2$. Therefore, results from sucrose-evoked EPSCs help exclude both excitability changes and changes in NMDA receptor function as explanations for the PDBu-induced recovery of $I_{\mathrm{NMDAR}}$.

We also performed similar experiments in mass cultures to ensure that activation of dormant synapses is not unique to autaptic synapses. In conventional mass cultures that form more complex and divergent synaptic interconnections, we examined sucrose responses as in the autaptic recordings in Figure 5A. Similar to autaptic responses, sucrose-evoked synaptic responses in mass culture were mildly but significantly potentiated after PDBu application (Fig. 5B). These observations from conventional synaptic connections suggest that the effects of PDBu are not confined to autaptic synapses.

In mass cultures, we also tested for PDBu-mediated recovery from dormancy using an NMDAR-based assay. We triggered 
transmitter release by incubating cells for $10 \mathrm{~min}$ in $200 \mathrm{~mm}$ sucrose in the presence of $10 \mu \mathrm{M}$ MK-801 (with NBQX and bicuculline present throughout). Because sucrose-induced release is not dependent on action potentials or calcium, our protocol helped ensure that even NMDARs at synapses that were infrequently activated by spontaneous activity would be blocked. After incubation, we measured synaptic NMDAR responses to spontaneous network activity. After MK-801 block, we never saw synaptic events within 150 210 s continual recording from any of four cells, even during elevated $\mathrm{K}^{+}$application to stimulate network activity and glutamate release $(n=4)$. By contrast, during $\mathrm{PDBu}$ application, synaptic NMDAR events became more evident (12 burst-like events in $150 \mathrm{~s}$ of recording from four cells) (supplemental Fig. S4, available at www.jneurosci.org as supplemental material). In all cells, we verified that these post-PDBu currents were sensitive to D-APV. The burst-like nature of the currents could be associated with NMDAR resurrection by $\mathrm{PDBu}$ throughout the network, with the long-lived NMDAR EPSPs promoting bursts of firing in presynaptic cells. Thus, this experiment is generally consistent with the idea that presynaptic dormancy also applies to more complex networks.

\section{Synaptic release properties and $\mathrm{PDBu}-$ mediated potentiation} Our results thus far exclude postsynaptic explanations for $\mathrm{PDBu}$ mediated revival of $I_{\text {NMDAR }}$ after synaptic MK-801 block and support the idea that PKC-independent presynaptic actions of $\mathrm{PDBu}$ recruit release from dormant terminals in simple and complex networks. In the literature, phorbol esters enhance vesicle release efficiency, increase synaptic $P_{\mathrm{r}}$, and hence increase synaptic strength (Malenka et al., 1986; Shapira et al., 1987; Basu et al., 2007). In many studies, the effect of phorbol ester on vesicular release efficiency is evaluated by paired-pulse modulation (Shapira et al., 1987; Muller et al., 1988; Yawo, 1999), or by the ratio of charge integral from an isolated evoked EPSC relative to the release from the entire release-ready vesicle pool (Basu et al., 2007; Wierda et al., 2007). These estimates, however, evaluate the conglomerate of changes in release from the entire population vesicles distributed across all synapses (Branco and Staras, 2009). Therefore, nothing is known about the synaptic $P_{\mathrm{r}}$ profile of the initially dormant synapses once their release activity is restored.

The $P_{\mathrm{r}}$ profiles of a population of synapses can be revealed by the kinetics of MK-801 progressive block of $I_{\mathrm{NMDAR}}$ (Hessler et al., 1993; Rosenmund et al., 1993). We used the kinetic changes in the progressive block after PDBu potentiation to probe changes of synaptic $P_{\mathrm{r}}$ distribution in the activated synapses after potentiation. We also examined the synaptic $P_{\mathrm{r}}$ profile from those basally quiescent terminals, once they were activated by $\mathrm{PDBu}$, with similar MK-801 progressive block.

In control cells, the decay of the first 25 peak NMDAR EPSCs during MK-801 progressive block was best described by a sum of two exponentials (Fig. 6A2, open circle), suggesting that synaptic transmission was composed of fast and slow components, which represent at least two populations of synapses with high and low $P_{\mathrm{r}}$ (Hessler et al., 1993). Note that this observation does not ex- clude the idea of a continuous distribution of synaptic $P_{\mathrm{r}}$ values (Rosenmund et al., 1993; Murthy et al., 1997).

PDBu treatment before any MK-801 application accelerated the subsequent MK-801 progressive block of the current (Fig. $6 A 1 ; A 2$, solid circle). Because we have previously ruled out the effect of PDBu on $P_{\mathrm{o}}$ of NMDARs (Fig. 2), the most plausible explanation for the faster EPSC block by MK-801 is that PDBu elevated $P_{\mathrm{r}}$ across synapses. The accelerated decay during progressive current block also yielded a greater proportion of the fast component compared with the control $(23.8 \pm 9.8$ vs $64 \pm 3 \%)$ (for full fit parameters, see Fig. 6A2, legend), suggesting that $\mathrm{PDBu}$ increased the average synaptic $P_{\mathrm{r}}$, as well as increasing the proportion of high $P_{\mathrm{r}}$ terminals (Rosenmund et al., 1993).

Blocking synaptic NMDARs at untreated synapses eliminated $I_{\text {NMDAR }}$ from synapses that respond to our stimulus protocol. After receptor blockade, PDBu promoted recovery of $I_{\mathrm{NMDAR}}$ as described previously. The MK-801 progressive block of PDBurecovered $I_{\text {NMDAR }}$ also consisted of biexponential components with significantly slower decay (Fig. 6A2, gray circle) compared with the basal, unblocked population of terminals that were potentiated by PDBu before progressive block. The slower decay of the EPSC block in the recovered current suggests that the recovered current arises from a subset of terminals that exhibited significantly lower synaptic $P_{\mathrm{r}}$ than the PDBu-potentiated population of initially active synapses.

When comparing the rate of progressive block, the contrast in synaptic $P_{\mathrm{r}}$ profiles between PDBu-recovered current (slow MK801 block) (Fig. 6 A2, gray) and the current that was derived from all synapses in the presence of PDBu (fast MK-801 block) (Fig. $6 \mathrm{~A}$, solid) suggests that $\mathrm{PDBu}$ did not potentiate synaptic output uniformly across terminals. Release from initially dormant synapses was restored by PDBu. However, this restoration was not accompanied by the high synaptic $P_{\mathrm{r}}$ profile and fast MK-801 block observed at initially active terminals potentiated by $\mathrm{PDBu}$. Instead, the MK- 801 block profile from the awakened terminals was closer to the slow MK- 801 block profile of naive active synapses before $\mathrm{PDBu}$ application (control, without PDBu) (Fig. $6 A 2$, open). The difference in synaptic $P_{\mathrm{r}}$ distributions induced by $\mathrm{PDBu}$ from initially active terminals versus quiescent termi- 
A1

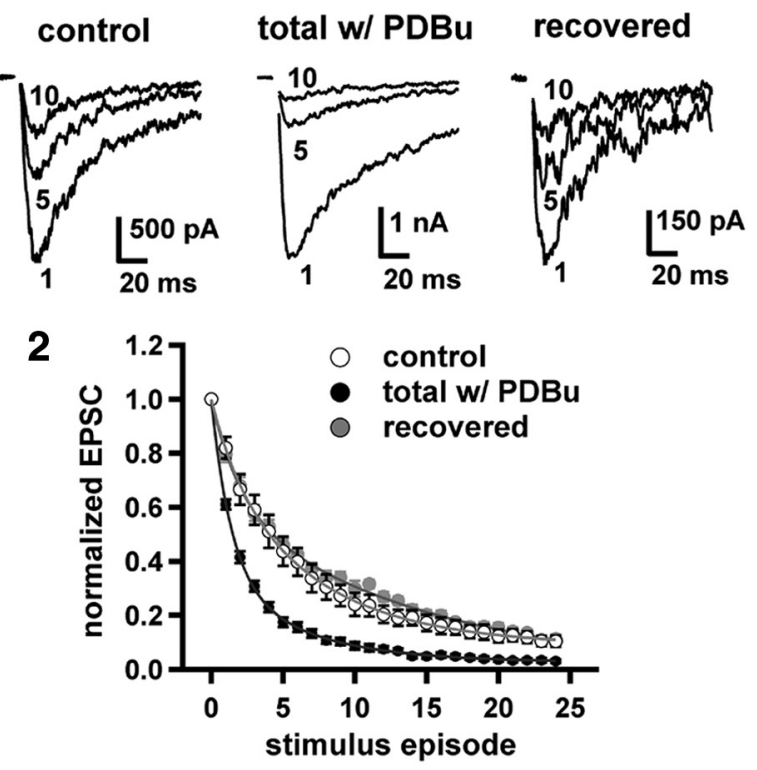

B1

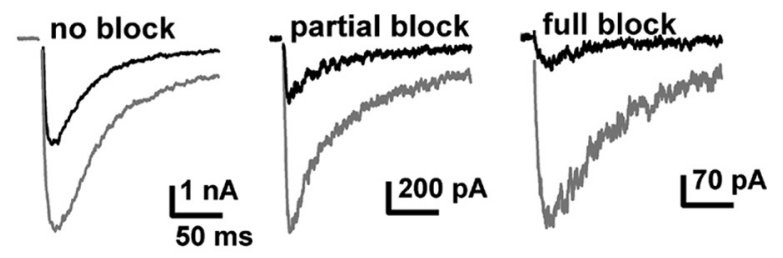

2

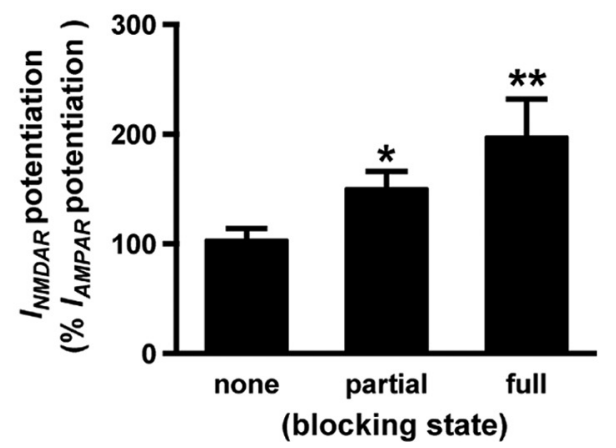

Figure 6. Synaptic $P_{r}$ differs at active versus dormant synapses after PDBu potentiation. $A$, PDBu-activated quiescent synapses show lower overall synaptic $P_{r}$. A1, Examples of graded depression of $I_{\text {NMDAR }}$ during MK-801 progressive block in control synapses (left), synapses that were potentiated by PDBu without previous MK-801 block (middle), and PDBu-recovered synapses (right). EPSCs from the indicated stimulus episodes in the presence of MK-801 are super-

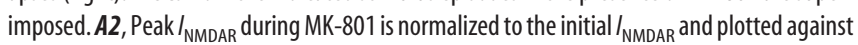
stimulus episode. The plot, averaged from eight to nine cells, is best fitted by a sum of two exponentials (gray lines) in all three conditions. The kinetics of progressive block (25 stimuli) has $\tau_{\text {fast }}=2.0 \pm 0.6(23.8 \pm 9.8 \%)$ and $\tau_{\text {slow }}=7.6 \pm 0.9(67.3 \pm 9.2 \%)$ in control (open; $n=$ 8), $\tau_{\text {fast }}=1.38 \pm 0.04(64 \pm 3 \%)$ and $\tau_{\text {slow }}=5.99 \pm 0.59(32 \pm 3 \%)$ in PDBu-potentiated current (solid; $n=9)$, and $\tau_{\text {fast }}=1.98 \pm 0.35(38 \pm 6 \%)$ and $\tau_{\text {slow }}=14.58 \pm 3.8(62.6 \pm$ $2 \%$ ) in PDBu-recovered current (gray; $n=8$ ). $B$, PDBu more strongly potentiated $I_{\text {NMDAR }}$ at synapse with low $P_{\mathrm{r}} \boldsymbol{B} 1$, Examples of PDBu-dependent potentiation from a neuron without receptor blockade (left), a neuron with synaptic $I_{\text {NMDAR }}$ that was partially blocked ( $\sim 15 \%$ of initial current; middle), and a neuron with synaptic $I_{\text {NMDAR }}$ that was fully blocked (right). The EPSCs after PDBu potentiation (gray) are superimposed on the EPSC before PDBu treatment (black). B2, Summary of PDBu-mediated potentiation in all three conditions. Level of $I_{\text {NMDAR }}$ potentiation by PDBu is normalized to $I_{\text {AMPAR }}$ potentiation from the same cells (no block, $n=$ 12; partial block, $n=10$; full block, $n=11 ; p<0.00003$ by one-way ANOVA; ${ }^{*} p<0.003$, compared with no block; ${ }^{* *} p<0.05$, compared with partial block by paired $t$ test). Error bars indicate SEM. nals may suggest that initial release competence of the terminal affects the nature of $\mathrm{PDBu}$ potentiation.

To test further whether the initial release properties of synapses affects their response to $\mathrm{PDBu}$, we examined whether $\mathrm{PDBu}$ differentially potentiates synaptic output from terminals with different $P_{\mathrm{r}}$. We reasoned that a single episode of brief depolarization should evoke transmitter release from the terminals of highest synaptic $P_{\mathrm{r}}$, the synaptic output from the high $P_{\mathrm{r}}$ terminals should dominate the isolated EPSC at baseline. Likewise, after partially (achieved with MK-801 during a limited number of stimulus trials) or fully blocking the synaptic $I_{\text {NMDAR }}$, the residual current should represent the synaptic output from the remaining low $P_{\mathrm{r}}$ terminals. If the initial $P_{\mathrm{r}}$ state affects synaptic response to $\mathrm{PDBu}$ potentiation, we would expect differential effects of $\mathrm{PDBu}$ potentiation on the residual current from partially or fully blocked cells compared with that in the control. We compared the level of $\mathrm{PDBu}$ potentiation in the control current, the residual current from partially blocked cells, and the residual current from fully blocked cells.

In the control cells without any preceding receptor block, $\mathrm{PDBu}$, as previously shown, increased baseline $I_{\mathrm{NMDAR}}$ approximately twofold (Fig. 6B1, left). Partial elimination of $I_{\text {NMDAR }}$ to $\sim 15 \%$ of the initial NMDAR-mediated current did not affect $I_{\mathrm{AMPAR}}\left(102 \pm 10 \%\right.$ of the initial $I_{\mathrm{AMPAR}}$ before MK-801 block; $n=10$ ). Interestingly, PDBu treatment in partially blocked cells resulted in approximately threefold potentiation from the residual $I_{\text {NMDAR }}$ (Fig. 6B1, middle). When compared with the cells in which the synaptic $I_{\text {NMDAR }}$ was fully eliminated, PDBu potentiation of the residual current was, as previously shown, close to fivefold (Fig. 6B1, right).

Because there is considerable variability in the degree of PDBu potentiation across cells, we used PDBu potentiation of AMPARmediated EPSCs as the internal potentiation reference and normalized the level of PDBu potentiation in $I_{\mathrm{NMDAR}}$ to $I_{\mathrm{AMPAR}}$ potentiation from the same cell. This normalization should represent the true $\mathrm{PDBu}$ potentiation of $I_{\mathrm{NMDAR}}$ from synapses with different $P_{\mathrm{r}}$. After normalizing to the level of $I_{\text {AMPAR }}$ potentiation, $\mathrm{PDBu}$ treatment in control, unblocked cells yielded the expected relative potentiation value near $100 \%$ (resulting from parallel NMDAR and AMPAR EPSC potentiation) (Fig. 6B2). When similar normalization was applied to the current potentiation from partially blocked and from fully blocked cells, the ratio of $I_{\mathrm{NMDAR}}$ versus $I_{\mathrm{AMPAR}}$ potentiation increased from $\sim 100 \%$ in control to $\sim 150 \%$ in the partially blocked cells and to $\sim 200 \%$ in fully blocked cells (Fig. 6 B2). The graded increases of PDBu potentiation in $I_{\mathrm{NMDAR}}$ suggest that PDBu more strongly potentiates low $P_{\mathrm{r}}$ terminals. Thus, the initial synaptic $P_{\mathrm{r}}$ may influence synaptic response to $\mathrm{PDBu}$ potentiation. This result, combined with the progressive MK-801 block experiment, is consistent with the idea that level of potentiation might be negatively correlated to the initial release activity (Stevens and Wang, 1994; Gerber et al., 2008).

\section{NR2B contribution of the recovered current does not differ from baseline}

The results thus far suggest that $\mathrm{PDBu}$ potentiated postblock $I_{\mathrm{NMDAR}}$ by presynaptic mechanisms; the recovered currents arise from a subset of dormant synapses, or synapses with very low $P_{\mathrm{r}}$, that are not recruited by $\mathrm{Ca}^{2+}$ or by FSK. Studies have suggested that synaptic NMDAR composition correlates with synaptic activity as well as with developmental stage. Synapses with lower functionality or immature (nascent) synapses possess greater NR2B subunit postsynaptically (Williams et al., 1993; Monyer et 
al., 1994; Kirson and Yaari, 1996; Tovar and Westbrook, 1999; Ehlers, 2003). Therefore, PDBu-recovered synapses may be dominated by NR2B-containing receptors. We used the NR2B-specific antagonist ifenprodil to probe NR2B contribution to the recovered $I_{\mathrm{NMDAR}}$. We compared the ifenprodil sensitivity of the initial $I_{\mathrm{NMDAR}}$ and of PDBu-recovered $I_{\mathrm{NMDAR}}$. In the control condition, the peak of the initial (untreated, unblocked) $I_{\text {NMDAR }}$ was reduced by $\sim 60 \%$ in the presence of ifenprodil ( 3 $\mu \mathrm{M})$ (Fig. 7A, left panel). After MK-801 block, PDBu-recovered $I_{\mathrm{NMDAR}}$ showed similar ifenprodil sensitivity (Fig. $7 A$, right panel), suggesting that $\mathrm{PDBu}$-recovered $I_{\text {NMDAR }}$ consisted of a similar NR2B contribution compared with the initial EPSC.

In addition, we also examined the deactivation kinetics of the initial and the recovered $I_{\text {NMDAR }}$. EPSCs mediated by NR2B-contaning receptors feature slower deactivation kinetics compared with EPSCs mediated by NR2A-containing receptors (Cull-Candy and Leszkiewicz, 2004). The deactivation current of NMDAR-mediated EPSC can be fitted by a sum of two exponentials, which yields a weighted $\tau=132 \pm 13 \mathrm{~ms}(n=6)$ in the initial current (Fig. 7B). In the recovered current, we found a slightly but not significantly slower decay with weighted $\tau=181 \pm 24 \mathrm{~ms}$ (Fig. $7 B$ ). Together, the pharmacological and kinetic results suggest that there is no significant difference in the level of NR2B component in the initial current and the recovered current. Thus, the synapses that give rise to the recovered current do not preferentially harbor NR2Bcontaining receptors.

PDBu promotes transmitter release from presynaptically silent synapses

Our results suggest that $\mathrm{PDBu}$ promotes synaptic output from synapses that contribute very little to baseline transmission. These synapses could represent synapses with very low $P_{\mathrm{r}}$ and/or synapses that are completely dormant. Work from our group and others has shown that a fraction of glutamate terminals appear to be completely dormant presynaptically (Rosenmund et al., 2002; Altrock et al., 2003; Moulder et al., 2004; Ting et al., 2007). These latent synapses are considered presynaptically inactive because they contain presynaptic vesicles, identified by immunoreactivity for vGluT-1 or other vesicle markers, but fail to detectably recycle vesicles, defined by FM1-43FX uptake on strong depolarization designed to trigger recycling of all release-competent vesicles. We next asked whether PDBu-recovered EPSCs represent, in part, restoration of synaptic activity at these presynaptically inactive terminals. We examined the percentage of inactive synapses from the cultures that were acutely exposed to FSK or to PDBu for 2 min. The synaptic activity of individual terminals was assessed by FM1-43FX labeling with hyperkalemic depolarization (45 mM $\left[\mathrm{K}^{+}\right]_{\mathrm{o}}$ for $2 \mathrm{~min}$ ) (Pyle et al., 2000; Harata et al., 2001); excitatory terminals were then identified by positive immunoreactivity for vGluT-1. Inactive excitatory synapses were those that were immunoreactive for vGluT-1 but lacking FM1-43FX labeling (i.e., failed to meet a threshold that exceeded background fluorescence
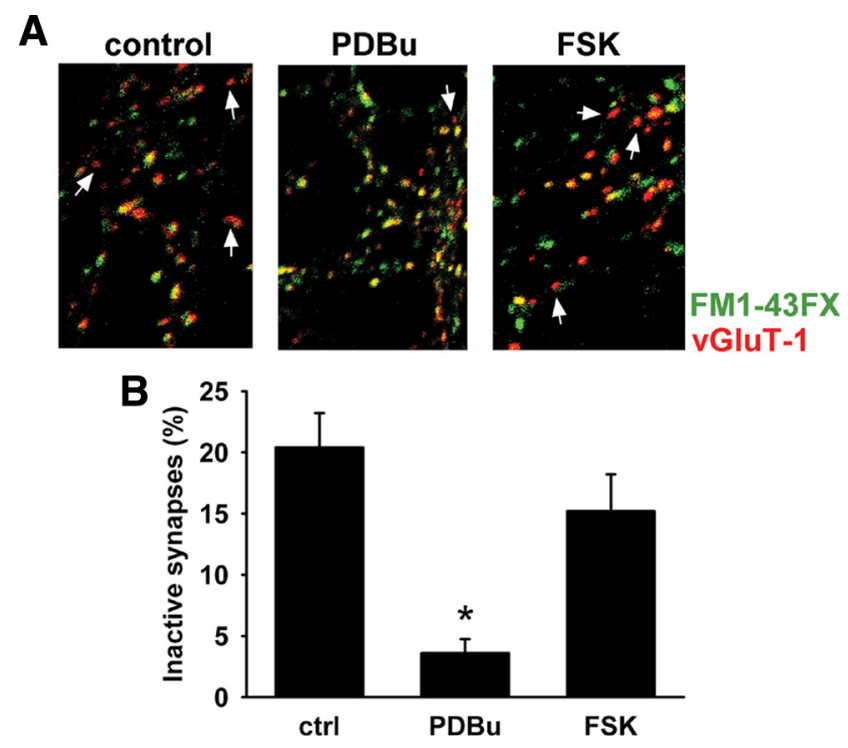

Figure 8. PDBu reduces the percentage of inactive excitatory synapses. $\boldsymbol{A}$, Example images from control (left), PDBu (middle)-, and FSK (right)-treated synapses. Active synapses were labeled with FM1-43FX (green); glutamatergic terminals were identified by immunoreactivity to vGluT-1 antisera (red). Puncta that are positive for vGluT-1 antisera but devoid of FM1-43FX labeling are inactive excitatory synapses (arrows). B, Quantification of the percentage of inactive glutamatergic terminals in control (20.4 $\pm 2.8 \%)$, or PDBu (3.6 $\pm 1.1 \%)$-, and FSK $(15.2 \pm 3 \%)$-treated synapses $\left({ }^{*} p<\right.$ 0.00005 compared with control; $n=5$ ). Summary results represent 250 puncta in each condition sampled in 50 fields from five independent experiments. Fields were selected and analyzed by an observer naive to experimental condition. Error bars indicate SEM.

intensity) (see Materials and Methods). In the control condition, the percentage of inactive synapses from the identified excitatory terminals was $\sim 20 \%$ (Fig. 8 A, left; $B$ ). Acute application of PDBu prominently reduced the percentage of inactive synapses (Fig. 
$8 A$, middle; $B$ ), indicating restored vesicle cycling at initially dormant terminals. In contrast to $\mathrm{PDBu}$, acute (2 min) application of FSK did not change the percentage of inactive synapses (Fig. $8 \mathrm{~A}$, right; $B)$.

We have previously used a fixed area threshold to identify inactive synapses, whereby active synapses must exceed a thresholded area of 10 pixels in the FM1-43 channel (Moulder et al., 2006, 2010). By this criterion, a somewhat higher percentage of inactive synapses was observed in all conditions, but similar comparative results were obtained $(27.2 \pm 3.0 \%$ control; $7.2 \pm 1.8 \%$ PDBu; $25.6 \pm 3.4 \%$ FSK).

Our previous work has shown that prolonged treatment $(4 \mathrm{~h})$ with FSK reduces the percentage of inactive synapses (Moulder et al., 2008). In sibling cultures of those represented in Figure 8, we repeated the experiments with $4 \mathrm{~h}$ FSK treatment and independently verified the reduction in dormant terminals with prolonged FSK treatment $(13.6 \pm 2.3 \%$ presynaptically inactive glutamatergic terminals compared with $27.2 \pm 2.9 \%$ in control; $n=5 ; p<0.0008)$. Therefore, the effects of prolonged activation of cAMP-dependent pathways are distinguishable from acute potentiation by FSK. In summary, acute treatment of PDBu, but not FSK, effectively activated transmitter release from the preexisting presynaptically silent synapses.

\section{PDBu potentiation in depolarization-silenced terminals}

We previously reported that increasing network activity by prolonged $(4 \mathrm{~h}$ ) depolarization with $35 \mathrm{~mm}$ extracellular potassium $\left(\left[\mathrm{K}^{+}\right]_{\mathrm{o}}\right)$ depresses synaptic output (Moulder et al., 2004). The synaptic output is depressed as a result of an increase in presynaptically silent synapses (Moulder et al., 2004). We next asked whether depolarization-inactivated presynaptic terminals, like the dormant synapses in baseline conditions, can be reactivated by $\mathrm{PDBu}$. If $\mathrm{PDBu}$ restores release from depolarizationinactivated terminals, we would expect a greater $I_{\mathrm{AMPAR}}$ potentiation and a greater $I_{\mathrm{NMDAR}}$ recovery in depolarized cultures, relative to the depressed baseline in these cultures. We depolarized neurons with $35 \mathrm{~mm}\left[\mathrm{~K}^{+}\right]_{\mathrm{o}}$ for $4 \mathrm{~h}$ and examined whether $\mathrm{PDBu}$ treatment more strongly potentiates $I_{\mathrm{AMPAR}}$ and more strongly recovers postblock $I_{\mathrm{NMDAR}}$. Depolarization reduced the average $I_{\mathrm{AMPAR}}$ by $70 \%$ (Fig. $9 A 1, A 2$ ), as previously reported (Moulder et al., 2004). In the depolarized neurons, PPR did not change while the evoked EPSC was depressed (Fig. 9A, legend), suggesting that vesicle $P_{\mathrm{r}}$ from the remaining active terminals did not change. These results are consistent with the idea of binary inactivation of terminals (Moulder et al., 2004). In depolarized neurons, $\mathrm{PDBu}$ treatment did not cause more potentiation of $I_{\text {AMPAR }}$ compared with the control (nondepolarized) (Fig. 9B). Similarly, PDBu resulted in a similar level of recovery in postblock $I_{\text {NMDAR }}$ (Fig. 9C). These results suggest that PDBu does not potentiate synaptic output from the synapses that are inactivated by prolonged depolarization.

\section{Discussion}

In this study, we used pharmacology, physiology, and activitydependent presynaptic labeling to probe the properties of quiescent (mute) synapses after synaptic potentiator treatment. These multiple approaches showed that presynaptically quiescent synapses are rapidly activated by PDBu, but not by FSK or by elevated extracellular $\mathrm{Ca}^{2+}$. Both basally functional and basally dormant, refractory terminals are potentiated by PDBu. After $\mathrm{PDBu}$ activation, initially quiescent terminals display heterogeneous synaptic $P_{\mathrm{r}}$ reminiscent of the $P_{\mathrm{r}}$ of basally active synapses. Quiescent synapses contain NMDA receptors pharmacologically
A1
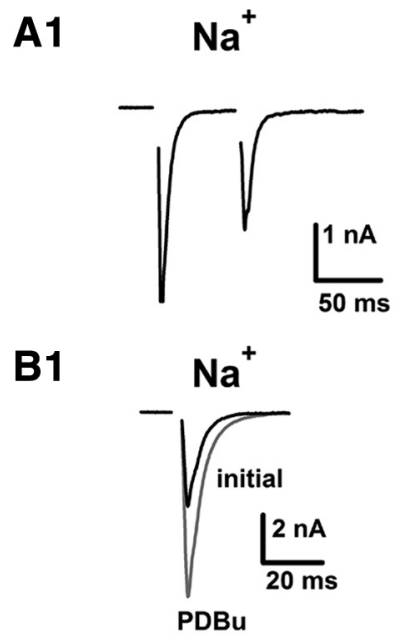

C1

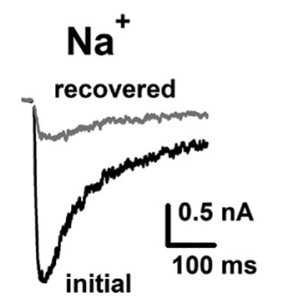

$2 \mathrm{~K}^{+}$

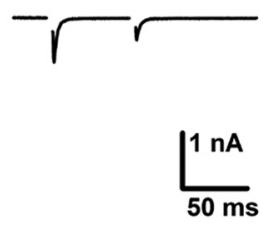

2

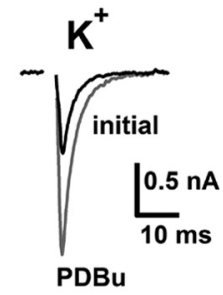

2

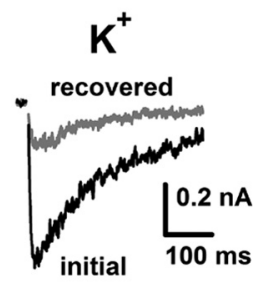

Figure 9. PDBu does not reactivate depolarization-inactivated synapses. $\boldsymbol{A}$, Prolonged depolarization (35 mm $\left[\mathrm{K}^{+}\right]_{0}$ for $4 \mathrm{~h}$ ) reduced synaptic current but did not affect PPR. Sample paired AMPAR-mediated EPSCs (50 ms interpulse interval) in control (A1) and in previously depolarized neurons (A2). EPSC measurements here and in other panels were all performed in physiological $\left[\mathrm{K}^{+}\right]_{0}(4 \mathrm{~mm})$ (see Materials and Methods). The average amplitude of $I_{\text {AMPAR }}$ was $3.80 \pm 0.94 \mathrm{nA}$ in control $\left(\mathrm{Na}^{+} ; n=9\right)$, and $1.07 \pm 0.34 \mathrm{nA}$ in depolarized neurons $\left(\mathrm{K}^{+} ; n=\right.$ $10 ; p<0.03)$. The paired-pulse ratio $\left(\right.$ EPSC $_{\text {second }} /$ EPSC $_{\text {first }}$ ) in control was $0.69 \pm 0.05(n=9)$ and in sibling depolarized neurons was $0.70 \pm 0.06(n=10 ; p=0.84) . B, I_{\text {AMPAR }}$ potentiation by PDBu was similar in control and in depolarized neurons. Examples of PDBu potentiated $I_{\text {AMPAR }}$ in control (B1) and in depolarized neurons (B2). Average PDBu potentiation in IMPAR $_{\text {in }}$ control was $264.3 \pm 70 \%$ relative to baseline $(n=9)$ and in depolarized cultures it was $266 \pm$ $37.7 \%$ relative to baseline $(n=10 ; p=0.98$ ). C, Sample traces of PDBu-mediated recovered $I_{\text {NMDAR }}$ in control (C1) and in depolarized neurons ( $(2)$. Recovered currents (gray) are superimposed on the initial currents (black). Average effect of PDBu on the recovered synaptic NMDAR $_{\text {in }}$ control was $21.6 \pm 6.1 \%$ relative to the initial current $(n=9)$ and in depolarized neurons it was $31.3 \pm 6.1 \%$ to the initial current $(n=10 ; p=0.27)$.

and kinetically indistinguishable from those of active synapses. Last, PDBu restores release at basally quiescent synapses, but not at depolarization-inactivated synapses. These observations highlight different mechanisms underlying seemingly similar second messenger-mediated presynaptic potentiation; whereas acute FSK-mediated potentiation mainly enhances synaptic output from the preexisting active terminals, $\mathrm{PDBu}$ increases $P_{\mathrm{r}}$ at the active terminals and restores release competence at basally dormant synapses. These observations may help to elucidate the roles of different signaling pathways in temporal or spatial changes of neuronal connectivity.

\section{Presynaptic causes of PDBu-mediated $I_{\mathrm{NMDAR}}$ recovery} Several alternative mechanisms could potentially cause the potentiation-associated revival of the synaptic $I_{\mathrm{NMDAR}}$ after MK801 block. First, transmitter could spill out from synapses as a result of transmission enhanced by conventional increases in vesicular $P_{\mathrm{r}}$. This transmitter overlap from active terminals may taint receptors not previously exposed to transmitter during MK801 application and could cause PDBu-induced recovery of $I_{\text {NMDAR }}$. Enhanced presynaptic release increases the likelihood of 
transmitter spillover, especially when glutamate transporter are compromised (Mennerick and Zorumski, 1995; Asztely et al., 1997; Diamond, 2001; Arnth-Jensen et al., 2002; Christie and Jahr, 2006). In the present study, however, neither of two additional presynaptic potentiators produced significant $I_{\text {NMDAR }}$ recovery, suggesting that increasing transmission alone, with any accompanying spillover, is not sufficient to cause current recovery by PDBu after MK-801 block.

A second alternative presynaptic explanation for $\mathrm{PDBu}$ induced recovery of $I_{\mathrm{NMDAR}}$ is that PDBu enhances excitability at axon branches where action potential invasion fails before PDBu incubation. Several lines of evidence argue against this possibility. First, artificially enhancing excitability does not recover $I_{\text {NMDAR }}$ (supplemental Fig. S1, available at www.jneurosci.org as supplemental material). Second, two experimental protocols designed to circumvent action potentials for evoking release, hypertonicityevoked EPSCs and hyperkalemia-induced FM1-43FX labeling, both suggest that terminals that do not initially participate in release are recruited by $\mathrm{PDBu}$.

A third alternative explanation for the effects of PDBu after MK-801 block is postsynaptic modification of NMDARs. Several studies showed that phorbol esters increase surface expression (Lan et al., 2001; Scott et al., 2001), alter gating properties (Durand et al., 1992; Lan et al., 2001; Lin et al., 2006), and enhance lateral movement (Fong et al., 2002; Groc et al., 2004) of NMDARs; inhibiting PKC activity abolishes the modification by phorbol ester of the receptors (Lan et al., 2001; Fong et al., 2002; Lin et al., 2006). Several experiments distinguish PDBudependent revival of $I_{\mathrm{NMDAR}}$ from postsynaptic receptor modification. First, with short treatment of PDBu, we failed to detect significant whole-cell or synaptic current recovery after blocking all surface receptors, despite robust presynaptic potentiation assessed by $I_{\mathrm{AMPAR}}$. Second, although not all presynaptic effects of PDBu are PKC dependent, the postsynaptic effects on NMDARs are PKC dependent (Durand et al., 1992; Lan et al., 2001; Scott et al., 2001; Fong et al., 2002; Lin et al., 2006). Preventing PKC activation in the presence of PDBu did not hamper $I_{\mathrm{NMDAR}}$ recovery. Third, the slower progressive NMDAR block of PDBurecovered $I_{\mathrm{NMDAR}}$ compared with $\mathrm{PDBu}$-speeded progressive block in active synapses excludes simple lateral movement of NMDARs into active terminals. Fourth, sucrose-evoked AMPAR EPSCs are potentiated by the amount expected from $I_{\text {NMDAR }}$ recovery experiments (Fig. 5). We cannot fully explain why this effect of PDBu has not been observed in all previous experiments (Basu et al., 2007; Wierda et al., 2007), although the small effect size $(25 \%)$ relative to effects on evoked EPSCs may partially explain the discrepancy. Finally, results from presynaptic, activitydependent FM dye labeling (Fig. 8) directly support a presynaptic explanation for PDBu-induced $I_{\mathrm{NMDAR}}$ recovery.

\section{Presynaptically dormant synapses}

Synapses with low functional connectivity, caused either presynaptically or postsynaptically, serve as a reservoir that increases synaptic malleability (Voronin and Cherubini, 2004; Kerchner and Nicoll, 2008). Presynaptically silent synapses occur in developing and mature hippocampal cultures (Tong et al., 1996; Ma et al., 1999), slices (Kullmann et al., 1996; Gasparini et al., 2000), spinal cord (Jack et al., 1981), and cultured sensory to motor neurons in Aplysia (Kim et al., 2003). In hippocampal cultures, release at quiescent synapses can be recruited by repetitive stimulation (Shen et al., 2006; Yao et al., 2006), application of the neurotrophic factor BDNF (Collin et al., 2001; Slutsky et al., 2004), or by prolonged activation of cAMP signaling (Ma et al.,
1999; Moulder et al., 2008), as opposed to the acute cAMP elevation in the present study. The neurotrophic or activity-dependent activation of dormant synapses may involve cytoskeletal reorganization at the presynaptic terminals, which facilitates the maturation of release apparatus (Slutsky et al., 2004; Shen et al., 2006; Yao et al., 2006).

The contrast between immediate actions of FSK and PDBu may reflect distinct downstream targets of the modulators. The need for slow, cAMP-dependent unsilencing pathways, nonetheless, makes the rapidity of PDBu unsilencing quite surprising and may suggest multiple routes to synaptic unsilencing. Our results show that the rapid unsilencing by $\mathrm{PDBu}$ is $\mathrm{PKC}$ independent. Because PKC-independent effects of phorbol esters involve the vesicle priming protein Munc13 (Betz et al., 1998; Rhee et al., 2002), PDBu-induced synaptic awakening likely involves Munc13-induced vesicle priming at dormant terminals.

Our previous work has demonstrated a form of activitydependent presynaptic adaptation of synaptic output; chronic depolarization presynaptically silences glutamate transmission (Moulder et al., 2004, 2006). Depolarization-silenced terminals are apparently distinct from basally quiescent terminals, as PDBu restores release at basally quiescent synapses but not at depolarization-silenced synapses. We have demonstrated this both with imaging (Moulder et al., 2008) and with electrophysiological assessments (present work). Although reduced levels of the priming protein Munc13-1 appears to be a commonality between basally silent and depolarization-silenced synapses (Jiang et al., 2010), other components contributing to release competence must differ to explain the selective PDBu sensitivity of basally dormant synapses. Therefore, there may be at least two partially independent molecular pathways that lead to presynaptic silencing. Additional study is required to delineate the molecular distinction between the two classes of presynaptically silent synapses.

\section{PDBu-mediated potentiation and synaptic $P_{\mathbf{r}}$}

An increased average $P_{\mathrm{r}}$ from a population of synapses can be achieved by increasing $P_{\mathrm{r}}$ at each terminal, and/or by increasing the proportion of high $P_{\mathrm{r}}$ terminals. According to the accelerated fast decay and the increased fast component in MK-801 progressive block after $\mathrm{PDBu}$ potentiation, $\mathrm{PDBu}$ seems to increase the proportion of high $P_{\mathrm{r}}$ terminals. This interpretation is consistent with the increases of vesicle turnover at active terminals by $\mathrm{PDBu}$ observed by others (Waters and Smith, 2000) and echoes the idea that PDBu acts on Munc13 to enhance fusion efficiency of vesicles (Basu et al., 2007). A similar synaptic $P_{\mathrm{r}}$ shift is observed with FSK treatment at the calyx of Held (Kaneko and Takahashi, 2004), suggesting that both FSK and PDBu increase synaptic $P_{\mathrm{r}}$ of functional terminals.

Interestingly, after PDBu potentiation, the synaptic $P_{\mathrm{r}}$ profile of initially quiescent synapses does not match the synaptic $P_{\mathrm{r}}$ profile of the entire population of $\mathrm{PDBu}$-potentiated synapses (Fig. 6A2). This could represent a deficiency in the quiescent synapses that renders these synapses unable to exhibit $\mathrm{PDBu}$ induced $P_{\mathrm{r}}$ potentiation. By this view, $\mathrm{PDBu}$ has two entirely independent effects. One effect increases synaptic $P_{\mathrm{r}}$ at already active synapses, and the other effect restores dormant synapses to an active state with a baseline synaptic $P_{\mathrm{r}}$ distribution. Alternatively, the dormant synapses represent synapses at the extreme low end of the synaptic $P_{\mathrm{r}}$ spectrum. By this view, the PDBu effect on the dormant synapses is an extension of the observation that the lowest $P_{\mathrm{r}}$ terminals are most sensitive to $\mathrm{PDBu}$ stimulation (Fig. $6 B$ ). This latter view is more parsimonious since it does not 
postulate different molecular mechanisms for the synaptic $P_{\mathrm{r}}$ increases and unsilencing effects. However, this interpretation is difficult to reconcile with the observations that elevated $\mathrm{Ca}^{2+}$, acute FSK treatment, and high potassium depolarization (during FM1-43FX labeling) are all ineffective in recruiting the PDBusensitive terminals containing low $P_{\mathrm{r}}$ vesicles.

Together, our observations provide evidence for differential potentiation by synaptic modulators and suggest that presynaptically dormant synapses can be rapidly recovered. Our results suggest multiple molecular and physiological pathways, including those that target vesicle priming, can trigger presynaptic unsilencing. Our studies also provide new insight into the multiple presynaptic actions of phorbol esters. Rapid awakening of dormant terminals is a form of plasticity that may be particularly well suited for altering the functional connectivity of neural networks.

\section{References}

Altrock WD, tom Dieck S, Sokolov M, Meyer AC, Sigler A, Brakebusch C, Fässler R, Richter K, Boeckers TM, Potschka H, Brandt C, Löscher W, Grimberg D, Dresbach T, Hempelmann A, Hassan H, Balschun D, Frey JU, Brandstätter JH, Garner CC, et al. (2003) Functional inactivation of a fraction of excitatory synapses in mice deficient for the active zone protein Bassoon. Neuron 37:787-800.

Arnth-Jensen N, Jabaudon D, Scanziani M (2002) Cooperation between independent hippocampal synapses is controlled by glutamate uptake. Nat Neurosci 5:325-331.

Asztely F, Erdemli G, Kullmann DM (1997) Extrasynaptic glutamate spillover in the hippocampus: dependence on temperature and the role of active glutamate uptake. Neuron 18:281-293.

Basu J, Betz A, Brose N, Rosenmund C (2007) Munc13-1 C1 domain activation lowers the energy barrier for synaptic vesicle fusion. J Neurosci 27:1200-1210.

Bekkers JM, Stevens CF (1989) NMDA and non-NMDA receptors are colocalized at individual excitatory synapses in cultured rat hippocampus. Nature 341:230-233.

Betz A, Ashery U, Rickmann M, Augustin I, Neher E, Südhof TC, Rettig J, Brose N (1998) Munc13-1 is a presynaptic phorbol ester receptor that enhances neurotransmitter release. Neuron 21:123-136.

Betz WJ, Bewick GS (1992) Optical analysis of synaptic vesicle recycling at the frog neuromuscular junction. Science 255:200-203.

Blumberg PM (1991) Complexities of the protein kinase C pathway. Mol Carcinog 4:339-344.

Branco T, Staras K (2009) The probability of neurotransmitter release: variability and feedback control at single synapses. Nat Rev Neurosci 10:373-383.

Brose N, Rosenmund C (2002) Move over protein kinase C, you've got company: alternative cellular effectors of diacylglycerol and phorbol esters. J Cell Sci 115:4399-4411.

Carroll RC, Nicoll RA, Malenka RC (1998) Effects of PKA and PKC on miniature excitatory postsynaptic currents in CAl pyramidal cells. J Neurophysiol 80:2797-2800.

Chang CY, Mennerick S (2010) Dynamic modulation of phasic and asynchronous glutamate release in hippocampal synapses. J Neurophysiol 103:392-401.

Chavis P, Mollard P, Bockaert J, Manzoni O (1998) Visualization of cyclic AMP-regulated presynaptic activity at cerebellar granule cells. Neuron 20:773-781.

Christie JM, Jahr CE (2006) Multivesicular release at Schaffer collateralCA1 hippocampal synapses. J Neurosci 26:210-216.

Collin C, Vicario-Abejon C, Rubio ME, Wenthold RJ, McKay RD, Segal M (2001) Neurotrophins act at presynaptic terminals to activate synapses among cultured hippocampal neurons. Eur J Neurosci 13:1273-1282.

Crawford DC, Moulder KL, Gereau RW 4th, Story GM, Mennerick S (2009) Comparative effects of heterologous TRPV1 and TRPM8 expression in rat hippocampal neurons. PLoS ONE 4:e8166.

Cull-Candy SG, Leszkiewicz DN (2004) Role of distinct NMDA receptor subtypes at central synapses. Sci STKE 2004:re16.

Diamond JS (2001) Neuronal glutamate transporters limit activation of NMDA receptors by neurotransmitter spillover on CA1 pyramidal cells. J Neurosci 21:8328-8338.
Durand GM, Gregor P, Zheng X, Bennett MV, Uhl GR, Zukin RS (1992) Cloning of an apparent splice variant of the rat $N$-methyl-D-aspartate receptor NMDAR1 with altered sensitivity to polyamines and activators of protein kinase C. Proc Natl Acad Sci U S A 89:9359-9363.

Ehlers MD (2003) Activity level controls postsynaptic composition and signaling via the ubiquitin-proteasome system. Nat Neurosci 6:231-242.

Fong DK, Rao A, Crump FT, Craig AM (2002) Rapid synaptic remodeling by protein kinase C: reciprocal translocation of NMDA receptors and calcium/calmodulin-dependent kinase II. J Neurosci 22:2153-2164.

Forsythe ID, Westbrook GL (1988) Slow excitatory postsynaptic currents mediated by $N$-methyl-D-aspartate receptors on cultured mouse central neurones. J Physiol 396:515-533.

Gasparini S, Saviane C, Voronin LL, Cherubini E (2000) Silent synapses in the developing hippocampus: Lack of functional AMPA receptors or low probability of glutamate release? Proc Natl Acad Sci U S A 97:9741-9746.

Gerber SH, Rah JC, Min SW, Liu X, de Wit H, Dulubova I, Meyer AC, Rizo J, Arancillo M, Hammer RE, Verhage M, Rosenmund C, Südhof TC (2008) Conformational switch of Syntaxin-1 controls synaptic vesicle fusion. Science 321:1507-1510.

Groc L, Heine M, Cognet L, Brickley K, Stephenson FA, Lounis B, Choquet D (2004) Differential activity-dependent regulation of the lateral mobilities of AMPA and NMDA receptors. Nat Neurosci 7:695-696.

Harata N, Ryan TA, Smith SJ, Buchanan J, Tsien RW (2001) Visualizing recycling synaptic vesicles in hippocampal neurons by FM1-43 photoconversion. Proc Natl Acad Sci U S A 98:12748-12753.

$\mathrm{He}$ Y, Zorumski CF, Mennerick S (2002) Contribution of presynaptic $\mathrm{Na}^{+}$ channel inactivation to paired-pulse synaptic depression in cultured hippocampal neurons. J Neurophysiol 87:925-936.

Hessler NA, Shirke AM, Malinow R (1993) The probability of transmitter release at a mammalian central synapse. Nature 366:569-572.

Hori T, Takai Y, Takahashi T (1999) Presynaptic mechanism for phorbol ester-induced synaptic potentiation. J Neurosci 19:7262-7267.

Huettner JE, Bean BP (1988) Block of N-methyl-D-aspartate-activated current by the anticonvulsant MK-801: selective binding to open channels. Proc Natl Acad Sci U S A 85:1307-1311.

Jack JJ, Redman SJ, Wong K (1981) Modifications to synaptic transmission at group Ia synapses on cat spinal motoneurones by 4 -aminopyridine. J Physiol 321:111-126.

Jahr CE (1992) High probability opening of NMDA receptor channels by L-glutamate. Science 255:470-472.

Jiang X, Litkowski PE, Taylor AA, Lin Y, Snider BJ, Moulder KL (2010) A role for the ubiquitin-proteasome system in activity-depedent presynaptc silencing. J Neurosci 30:1798-1809.

Kaneko M, Takahashi T (2004) Presynaptic mechanism underlying cAMPdependent synaptic potentiation. J Neurosci 24:5202-5208.

Kerchner GA, Nicoll RA (2008) Silent synapses and the emergence of a postsynaptic mechanism for LTP. Nat Rev Neurosci 9:813-825.

Kim JH, Udo H, Li HL, Youn TY, Chen M, Kandel ER, Bailey CH (2003) Presynaptic activation of silent synapses and growth of new synapses contribute to intermediate and long-term facilitation in Aplysia. Neuron 40:151-165.

Kirson ED, Yaari Y (1996) Synaptic NMDA receptors in developing mouse hippocampal neurones: functional properties and sensitivity to ifenprodil. J Physiol 497:437-455.

Kullmann DM, Erdemli G, Asztély F (1996) LTP of AMPA and NMDA receptor-mediated signals: evidence for presynaptic expression and extrasynaptic glutamate spill-over. Neuron 17:461-474.

Kutsuwada T, Kashiwabuchi N, Mori H, Sakimura K, Kushiya E, Araki K, Meguro H, Masaki H, Kumanishi T, Arakawa M, Mishina M (1992) Molecular diversity of the NMDA receptor channel. Nature 358:36-41.

Lan JY, Skeberdis VA, Jover T, Grooms SY, Lin Y, Araneda RC, Zheng X, Bennett MV, Zukin RS (2001) Protein kinase C modulates NMDA receptor trafficking and gating. Nat Neurosci 4:382-390.

Lin Y, Jover-Mengual T, Wong J, Bennett MV, Zukin RS (2006) PSD-95 and PKC converge in regulating NMDA receptor trafficking and gating. Proc Natl Acad Sci U S A 103:19902-19907.

Lonart G, Janz R, Johnson KM, Südhof TC (1998) Mechanism of action of rab3A in mossy fiber LTP. Neuron 21:1141-1150.

Lou X, Korogod N, Brose N, Schneggenburger R (2008) Phorbol esters modulate spontaneous and $\mathrm{Ca}^{2+}$-evoked transmitter release via acting on both Munc13 and protein kinase C. J Neurosci 28:8257-8267.

Lu WY, Jackson MF, Bai D, Orser BA, MacDonald JF (2000) In CA1 pyra- 
midal neurons of the hippocampus protein kinase $\mathrm{C}$ regulates calciumdependent inactivation of NMDA receptors. J Neurosci 20:4452-4461.

Ma L, Zablow L, Kandel ER, Siegelbaum SA (1999) Cyclic AMP induces functional presynaptic boutons in hippocampal CA3-CA1 neuronal cultures. Nat Neurosci 2:24-30.

Malenka RC, Madison DV, Nicoll RA (1986) Potentiation of synaptic transmission in the hippocampus by phorbol esters. Nature 321:175-177.

Mennerick S, Zorumski CF (1995) Presynaptic influence on the time course of fast excitatory synaptic currents in cultured hippocampal cells. J Neurosci 15:3178-3192.

Mennerick S, Que J, Benz A, Zorumski CF (1995) Passive and synaptic properties of hippocampal neurons grown in microcultures and in mass cultures. J Neurophysiol 73:320-332.

Monyer H, Burnashev N, Laurie DJ, Sakmann B, Seeburg PH (1994) Developmental and regional expression in the rat brain and functional properties of four NMDA receptors. Neuron 12:529-540.

Moulder KL, Meeks JP, Shute AA, Hamilton CK, de Erausquin G, Mennerick S (2004) Plastic elimination of functional glutamate release sites by depolarization. Neuron 42:423-435.

Moulder KL, Jiang X, Taylor AA, Olney JW, Mennerick S (2006) Physiological activity depresses synaptic function through an effect on vesicle priming. J Neurosci 26:6618-6626.

Moulder KL, Jiang X, Taylor AA, Shin W, Gillis KD, Mennerick S (2007) Vesicle pool heterogeneity at hippocampal glutamate and GABA synapses. J Neurosci 27:9846-9854.

Moulder KL, Jiang X, Chang C, Taylor AA, Benz AM, Conti AC, Muglia LJ, MennerickS (2008) A specific role for $\mathrm{Ca}^{2+}$-dependent adenylyl cyclases in recovery from adaptive presynaptic silencing. J Neurosci 28:5159-5168.

Moulder KL, Jiang X, Taylor AA, Benz AM, Mennerick S (2010) Presynaptically silent synapses studied with light microscopy. J Vis Exp pii:1676.

Muller D, Turnbull J, Baudry M, Lynch G (1988) Phorbol ester-induced synaptic facilitation is different than long-term potentiation. Proc Natl Acad Sci U S A 85:6997-7000.

Murthy VN, Sejnowski TJ, Stevens CF (1997) Heterogeneous release properties of visualized individual hippocampal synapses. Neuron 18:599-612.

Myme CI, Sugino K, Turrigiano GG, Nelson SB (2003) The NMDA-toAMPA ratio at synapses onto layer $2 / 3$ pyramidal neurons is conserved across prefrontal and visual cortices. J Neurophysiol 90:771-779.

Perkel DJ, Nicoll RA (1993) Evidence for all-or-none regulation of neurotransmitter release: implications for long-term potentiation. J Physiol 471:481-500.

Pyle JL, Kavalali ET, Piedras-Rentería ES, Tsien RW (2000) Rapid reuse of readily releasable pool vesicles at hippocampal synapses. Neuron 28:221-231.

Rhee JS, Betz A, Pyott S, Reim K, Varoqueaux F, Augustin I, Hesse D, Südhof TC, Takahashi M, Rosenmund C, Brose N (2002) $\beta$ Phorbol ester- and diacylglycerol-induced augmentation of transmitter release is mediated by Munc13s and not by PKCs. Cell 108:121-133.

Rosenmund C, Stevens CF (1996) Definition of the readily releasable pool of vesicles at hippocampal synapses. Neuron 16:1197-1207.

Rosenmund C, Clements JD, Westbrook GL (1993) Nonuniform probability of glutamate release at a hippocampal synapse. Science 262:754-757.

Rosenmund C, Sigler A, Augustin I, Reim K, Brose N, Rhee JS (2002) Differential control of vesicle priming and short-term plasticity by Munc13 isoforms. Neuron 33:411-424.

Schikorski T, Stevens CF (2001) Morphological correlates of functionally defined synaptic vesicle populations. Nat Neurosci 4:391-395.

Scott DB, Blanpied TA, Swanson GT, Zhang C, Ehlers MD (2001) An NMDA receptor ER retention signal regulated by phosphorylation and alternative splicing. J Neurosci 21:3063-3072.

Searl TJ, Silinsky EM (1998) Increases in acetylcholine release produced by phorbol esters are not mediated by protein kinase $\mathrm{C}$ at motor nerve endings. J Pharmacol Exp Ther 285:247-251.

Shapira R, Silberberg SD, Ginsburg S, Rahamimoff R (1987) Activation of protein kinase $C$ augments evoked transmitter release. Nature 325:58-60

Shen W, Wu B, Zhang Z, Dou Y, Rao ZR, Chen YR, Duan S (2006) Activityinduced rapid synaptic maturation mediated by presynaptic Cdc42 signaling. Neuron 50:401-414.

Silinsky EM, Searl TJ (2003) Phorbol esters and neurotransmitter release: more than just protein kinase C? Br J Pharmacol 138:1191-1201.

Slutsky I, Sadeghpour S, Li B, Liu G (2004) Enhancement of synaptic plasticity through chronically reduced $\mathrm{Ca}^{2+}$ flux during uncorrelated activity. Neuron 44:835-849.

Stevens CF, Sullivan JM (1998) Regulation of the readily releasable vesicle pool by protein kinase C. Neuron 21:885-893.

Stevens CF, Wang Y (1994) Changes in reliability of synaptic function as a mechanism for plasticity. Nature 371:704-707.

Ting JT, Kelley BG, Lambert TJ, Cook DG, Sullivan JM (2007) Amyloid precursor protein overexpression depresses excitatory transmission through both presynaptic and postsynaptic mechanisms. Proc Natl Acad Sci U S A 104:353-358.

Tong G, Jahr CE (1994) Multivesicular release from excitatory synapses of cultured hippocampal neurons. Neuron 12:51-59.

Tong G, Malenka RC, Nicoll RA (1996) Long-term potentiation in cultures of single hippocampal granule cells: a presynaptic form of plasticity. Neuron 16:1147-1157.

Tovar KR, Westbrook GL (1999) The incorporation of NMDA receptors with a distinct subunit composition at nascent hippocampal synapses in vitro. J Neurosci 19:4180-4188.

Tovar KR, Westbrook GL (2002) Mobile NMDA receptors at hippocampal synapses. Neuron 34:255-264.

Trudeau LE, Emery DG, Haydon PG (1996) Direct modulation of the secretory machinery underlies PKA-dependent synaptic facilitation in hippocampal neurons. Neuron 17:789-797.

Tsien JZ, Huerta PT, Tonegawa S (1996) The essential role of hippocampal CA1 NMDA receptor-dependent synaptic plasticity in spatial memory. Cell 87:1327-1338.

Voronin LL, Cherubini E (2004) "Deaf, mute and whispering" silent synapses: their role in synaptic plasticity. J Physiol 557:3-12.

Waters J, Smith SJ (2000) Phorbol esters potentiate evoked and spontaneous release by different presynaptic mechanisms. J Neurosci 20:7863-7870.

Weisskopf MG, Castillo PE, Zalutsky RA, Nicoll RA (1994) Mediation of hippocampal mossy fiber long-term potentiation by cyclic AMP. Science 265:1878-1882.

Wierda KD, Toonen RF, de Wit H, Brussaard AB, Verhage M (2007) Interdependence of PKC-dependent and PKC-independent pathways for presynaptic plasticity. Neuron 54:275-290.

Williams K, Russell SL, Shen YM, Molinoff PB (1993) Developmental switch in the expression of NMDA receptors occurs in vivo and in vitro. Neuron 10:267-278.

Xiong ZG, Raouf R, Lu WY, Wang LY, Orser BA, Dudek EM, Browning MD, MacDonald JF (1998) Regulation of $N$-methyl-D-aspartate receptor function by constitutively active protein kinase C. Mol Pharmacol 54:1055-1063.

Yao J, Qi J, Chen G (2006) Actin-dependent activation of presynaptic silent synapses contributes to long-term synaptic plasticity in developing hippocampal neurons. J Neurosci 26:8137-8147.

Yawo H (1999) Protein kinase C potentiates transmitter release from the chick ciliary presynaptic terminal by increasing the exocytotic fusion probability. J Physiol 515:169-180.

Zamponi GW, Bourinet E, Nelson D, Nargeot J, Snutch TP (1997) Crosstalk between $\mathrm{G}$ proteins and protein kinase $\mathrm{C}$ mediated by the calcium channel $\alpha 1$ subunit. Nature 385:442-446. 